\title{
Loss of KCNQ2 or KCNQ3 Leads to Multifocal Time-Varying Activity in the Neonatal Forebrain Ex Vivo
}

\author{
Bowen Hou, ${ }^{1}$ Nissi Varghese, ${ }^{1}$ Heun Soh, ${ }^{1}$ Sabato Santaniello, ${ }^{2}$ and ${ }^{\circledR}$ Anastasios V. Tzingounis ${ }^{1,2}$
}

https://doi.org/10.1523/ENEURO.0024-21.2021

${ }^{1}$ Department of Physiology and Neurobiology, University of Connecticut, Storrs, CT 06269 and ${ }^{2}$ Department of Biomedical Engineering, University of Connecticut, Storrs, CT 06269

\begin{abstract}
Epileptic encephalopathies represent a group of disorders often characterized by refractory seizures, regression in cognitive development, and typically poor prognosis. Dysfunction of KCNQ2 and KCNQ3 channels has emerged as a major cause of neonatal epilepsy. However, our understanding of the cellular mechanisms that may both explain the origins of epilepsy and inform treatment strategies for KCNQ2 and KCNQ3 dysfunction is still lacking. Here, using mesoscale calcium imaging and pharmacology, we demonstrate that in mouse neonatal brain slices, conditional loss of Kcnq2 from forebrain excitatory neurons (Pyr:Kcnq2 mice) or constitutive deletion of Kcnq3 leads to sprawling hyperactivity across the neocortex. Surprisingly, the generation of timevarying hypersynchrony in slices from Pyr:Kcnq2 mice does not require fast synaptic transmission. This is in contrast to control littermates and constitutive Kcnq3 knock-out mice where activity is primarily driven by fast synaptic transmission in the neocortex. Unlike in the neocortex, hypersynchronous activity in the hippocampal formation from Kcnq2 conditional and Kcnq3 constitutive knock-out mice persists in the presence of synaptic transmission blockers. Thus, we propose that loss of KCNQ2 or KCNQ3 function differentially leads to network hyperactivity across the forebrain in a region-specific and macro-circuit-specific manner.
\end{abstract}

Key words: channelopathy; epilepsy; KCNQ2; KCNQ3; neurodevelopmental disorders; neurology

\section{Significance Statement}

Neocortical hypersynchrony is a hallmark of neonatal epilepsy but its cellular mechanisms are unclear. This study shows that hypersynchrony in the neocortex can stem from the loss of KCNQ2 function in excitatory neurons even in the absence of fast synaptic transmission, unlike the hypersynchrony in response to KCNQ3 loss in the neocortex. This points to unique network dysfunctions involving potassium KCNQ2 channels as a mechanism for neonatal epilepsy.

\section{Introduction}

Ion channelopathies have been associated with multiple neurodevelopmental disorders over the last decades (Satterstrom et al., 2020; Wang et al., 2020). The KCNQ family, otherwise known as the Kv7 family, is a potassium channel family that has been propelled at the forefront of neurodevelopmental disorders (Geisheker et al., 2017; Cornet et al., 2018). Several of its members (KCNQ2-

Received January 19, 2021; accepted April 8, 2021; First published April 15, 2021.

The authors declare no competing financial interests.
KCNQ5) are expressed in the brain, across multiple brain regions and cell types (Greene and Hoshi, 2017). KCNQ2 channels were the second members of this family to be identified, with a rich history relating to neurodevelopmental disorders. KCNQ2 was first discovered over 20 years ago as a gene likely to cause benign familial neonatal seizures, a form of a self-limiting pediatric epilepsy disorder (Jentsch, 2000). However, since 2012, multiple

Author contributions: B.H., S.S., and A.V.T. designed research; B.H., N.V., and H.S. performed research; B.H., S.S., and A.V.T. analyzed data; S.S. and A.V.T. wrote the paper. 
studies have shown that KCNQ2 variants may lead to a large spectrum of symptoms and disorders, including Ohtahara syndrome and autism spectrum disorders (Cornet et al., 2018). These studies have demonstrated that KCNQ2 pathogenicity leads to developmental and epileptic encephalopathies (DEEs).

Over the years, our knowledge regarding KCNQ2 channel function in the brain has grown exponentially. This understanding has primarily arisen from the use of Kcnq2 transgenic mice and pharmacological agents that generally target KCNQ2-containing channels. KCNQ2 channels partner with KCNQ3 channels to form heteromeric channels containing two KCNQ2 and two KCNQ3 channels, classically referred to as the M-channels, as they mediate the M-current, a ubiquitous potassium conductance discovered in the 1980s (Jentsch, 2000). KCNQ2/3 channels are strategically located at sites of action potential initiation and regeneration, allowing them to control neuronal excitability by regulating the resting membrane potential of the axon initial segment and axons by preventing excessive firing (Cooper, 2011). Indeed, Kcnq2 deletion or expression of KCNQ2 pathogenic variants in neurons leads to elevated excitability, typically manifested as a higher number of action potentials and reduced spike frequency adaptation. Such changes in excitability occur in multiple brain regions, including the hippocampus and neocortex, and across multiple cell types (Peters et al., 2005; Singh et al., 2008; Soh et al., 2014, 2018; Verneuil et al., 2020).

To date, most studies on KCNQ2 and KCNQ3 channels using transgenic mice have focused on excitatory neurons in juvenile and adult mice. However, KCNQ2 channels are expressed early in development, with the mRNA expressed before birth (Kanaumi et al., 2008). Indeed, application of the pan-KCNQ blockers XE991 or linopirdine in brain slices from neonatal mice leads to increased excitability, providing evidence that KCNQ channel expression early in life prevents runaway excitation (Okada et al., 2003; Qiu et al., 2007). Additionally, overexpression of dominant-negative KCNQ2 channels in the brain during only the first of week of life results in seizures and premature lethality, suggesting that KCNQ2 channel expression is paramount during the neonatal period (Peters et al., 2005).

Loss-of-function KCNQ2 variants lead to severe neurodevelopmental disorders that present soon after birth. However, the manner in which KCNQ2 dysfunction alters cellular and network excitability early in development is currently unknown. Importantly, it remains unclear how

This work was supported by National Institutes of Health Grants R01 NS101596, HL137094, NS108874 (to A.V.T.) and the National Science Foundation CAREER Award 1845348 (to S.S).

Acknowledgements: We thank all members of the Tzingounis lab for discussions.

Correspondence should be addressed to Anastasios V. Tzingounis at anastasios.tzingounis@uconn.edu or Sabato Santaniello at sabato. santaniello@uconn.edu.

https://doi.org/10.1523/ENEURO.0024-21.2021

Copyright (C) 2021 Hou et al.

This is an open-access article distributed under the terms of the Creative Commons Attribution 4.0 International license, which permits unrestricted use, distribution and reproduction in any medium provided that the original work is properly attributed. the loss of KCNQ2 channels from excitatory neurons regulates developing hippocampal and neocortical circuits. To capture the dynamics and population activity across the forebrain in the presence and absence of KCNQ2 channels from excitatory neurons, we applied wide-field mesoscale calcium imaging from Kcnq2 conditional knock-out mice (Pyr:Kcnq2) and compared them with constitutive Kcnq3 knock-out mice. This approach allowed us to image the network dynamics of different forebrain circuits. We found that deletion of Kcnq2 or Kcnq3 resulted in multifocal and time-varying excitation across the forebrain. The spontaneous neuronal activity detected in Pyr:Kcnq2 slices was limited by ongoing GABAA receptor activity and amplified by fast glutamatergic transmission. Importantly, in slices from Pyr: Kcnq2 mice low-frequency (LF) activity persisted in the absence of fast synaptic transmission in the neocortex, unlike in slices from control or Kcnq3-null mice. Thus, ablation of Kcnq2 channels from excitatory neurons leads to distinct aberrant activity with a unique pharmacological profile.

\section{Materials and Methods}

\section{Animals}

All experiments were performed according to the guidelines described in the National Institutes of Health Guide for the Care and Use of Laboratory Animals and were approved by the Institutional Animal Care and Use Committee of the University of Connecticut.

All experimental procedures were performed on mice kept under a 14/10 h light/dark cycle with access to food and water ad libitum. Kcnq2 ${ }^{f / f}$ mice on a C57BL/6J background were previously developed and described by our group and others (Soh et al., 2014; Carver and Shapiro, 2019). In the presence of Cre recombinase, exons $2-4$ of the $K c n q 2^{f / f}$ mice are excised, leading to a premature stop codon and a non-functional protein. C57BL/6J (RRID:IMSR_JAX:000664), B6;129S6Polr2a ${ }^{T n(p b-C A G-G C a M P 5 g,-t d T o m a t o) T v r d} / \mathrm{J}$ (RRID:IMSR JAX:024477| PC-G5-tdT), and B6.129S2-Emx1 ${ }^{\text {tm1(cre)Krj } / \bar{J}}$ (RRID:IMSR_JAX:005628| Emx1-Cre), were purchased from The Jackson Laboratory. Breeding was performed inhouse at the University of Connecticut. For this study, we bred and developed Emx1-cre::PC-G5-tdT::Kcnq2 $2^{f /+}$ mice. We intercrossed these mice to obtain Emx1-cre::PCG5-tdT::Kcnq2 ${ }^{+/+}$, Emx1-cre::PC-G5-tdT::Kcnq2 ${ }^{f /+}$, and Emx1-cre:::PC-G5-tdT::Kcnq2 $2^{f / f}$ mice. For our experiments, we used either Emx1-cre::PC-G5-tdT::Kcnq2 $2^{+/+}$or Emx1cre::PC-G5-tdT::Kcnq2 $2^{f / f}$ mice. td-Tomato was used to verify that Cre expression was restricted to the forebrain. Annually we refresh the Cre-deleter line by purchasing new Emx1-cre breeding pairs and breeding them with a new set of mice carrying Kcnq2 $2^{f / f}$ and PC-G5-tdT. For Emx1-cre:: PC-G5-tdT::Kcnq3 ${ }^{-1-}$ we followed a similar strategy as with Emx1-cre::PC-G5-tdT::Kcnq2 $2^{f / f}$ line. Kcnq3 ${ }^{-1-}$ mice were kept on a C57BL/6J background. Genotypes were confirmed with PCR. Experiments were performed on mice of either sex.

\section{Acute slice preparation}

Mice between postnatal day (P)4 and P7 were brought from the vivarium into a designated area of the laboratory. 
Mice were then anesthetized using isoflurane. After verifying the mice were fully anesthetized, we rapidly decapitated them. Then, we placed the brains in ice-cold cutting solution containing the following: $26 \mathrm{~mm} \mathrm{NaHCO} 3,210 \mathrm{~mm}$ sucrose, $10 \mathrm{~mm}$ glucose, $2.5 \mathrm{~mm} \mathrm{KCl}, 1.25 \mathrm{~mm} \mathrm{NaH}_{2} \mathrm{PO}_{4}$, $0.5 \mathrm{mM} \mathrm{CaCl}_{2}$, and $7 \mathrm{~mm} \mathrm{MgCl}_{2}$. We mounted the brains on a vibratome (Leica, VS2000) and prepared $300 \mu \mathrm{m}$ horizontal slices. Slices were then placed in a $35-37^{\circ} \mathrm{C}$ holding chamber for $30 \mathrm{~min}$, and then moved to room temperature. The holding solution, which was the same as the recording solutions for the imaging and electrophysiological experiments, contained: $125 \mathrm{~mm} \mathrm{NaCl}, 26$ $\mathrm{mm} \mathrm{NaHCO} 3,2.5 \mathrm{~mm} \mathrm{KCl,} 1 \mathrm{~mm} \mathrm{NaH} \mathrm{PO}_{4}, 1.3 \mathrm{~mm} \mathrm{MgCl}$, $1.5 \mathrm{~mm} \mathrm{CaCl}_{2}$, and $12 \mathrm{~mm}$ D-glucose; $32-34^{\circ} \mathrm{C}, \sim 310$ mOsm. Slices were left to rest at room temperature for at least $1 \mathrm{~h}$ before experiments. All solutions were continuously bubbled with $5 \% \mathrm{CO}_{2} / 95 \% \mathrm{O}_{2}$. The slicing procedure was the same for whole-cell patch-clamp electrophysiology and calcium sensor imaging.

\section{Whole-cell patch-clamp electrophysiology}

For electrophysiological recordings, we used an upright BX-51W Olympus microscope (Olympus). Neurons were visualized using the built-in Olympus microscope Nomarski optics, allowing for differential interference contrast. We recorded from pyramidal neurons of the CA3 region of the hippocampus. For current-clamp recordings we used borosilicate glass capillaries (WPI, TW150F-3). The pipette resistance was 3-4 $M \Omega$ when filled with a solution containing the following: $130 \mathrm{~mm}$ potassium methylsulfate, $10 \mathrm{~mm} \mathrm{KCl,} 4$ mм NaCl, 4 mм Mg.ATP, 0.4 mм Na 4 .GTP, 10 mм HEPES, and $5 \mathrm{~mm}$ Tris-phosphocreatine (osmolarity $\sim 300-310$ mOsm). The $\mathrm{pH}$ was adjusted to $7.2-7.3$ with $\mathrm{KOH}$. Data were not corrected for the junction potential. All electrophysiological data were acquired using Multiclamp 700B amplifiers (Molecular Devices; RRID:SCR_018455) under the control of Clampex 10 software (Molecular Devices; RRID: SCR_011323). Data were acquired at $50 \mathrm{kHz}$ and low-pass filtered at $10 \mathrm{kHz}$. For current-clamp recordings, the bridge balance circuit built into the Multiclamp 700B was engaged to correct for series resistance.

\section{Imaging acute slices}

Optical imaging was performed on a Zeiss Axiozoom V.16 (Zeiss) using a PlanNeoFluar Z 1×, 0.25 NA objective with a $56 \mathrm{~mm}$ working distance. Images were recorded using an sCMOS camera (pco.edge 4.2) at a $500 \times 500$ pixel resolution. Image acquisition was performed using Micro-Manager $2.0 \quad \beta \quad$ (https://micro-manager.org/). Slices were submerged in a Warner chamber continuously perfused with extracellular solution $\left(32-34^{\circ} \mathrm{C}\right)$.

\section{$\Delta \mathrm{F} / \mathrm{F}$ calculations for two-dimensional (2D) plots}

We acquired and imported 300-s sCMOS sensor-recorded fluorescence time series (sampling rate: $F_{s}=20$ frames/s, 6000 frames/recording) in MATLAB (rel. 2019b; RRID:SCR_001622). Frames $(500 \times 500$ pixels, interpixel distance: $6 \mu \mathrm{m}$ ) were inspected for the external edges of the area covered by each hemisphere, and only pixels within the area delineated by these lines were further considered. Pixels within this area were divided into $N$ regions of interest (ROIs) using a simple linear iterative clustering (SLIC) algorithm (Achanta et al., 2012; MATLAB function: superpixels). The SLIC algorithm was applied to the first frame of each imported time series after foreground saturation to minimize the effects of spurious calcium activity at the beginning of the time series, and the value $N$ was optimized by the algorithm using an unsupervised strategy. This resulted in ROls that were spatially homogeneous, with pixels in each ROI showing similar fluorescence intensities. For any ROI $n(n=1,2, \ldots, N)$ and frame $k$ (i.e., $k=1,2,3, \ldots, 6000$ ), the ROI intensity, $F_{n}(k)$, was estimated as the average value of the fluorescence intensity across all pixels in the ROI, and the series of variations in fluorescence intensity, $\Delta F_{n} / F$, was estimated according to the following formula:

$$
\frac{\Delta F_{n}}{F}(k) \triangleq \frac{F_{n}(k)-\bar{F}(n, k)}{\bar{F}(n, k)},
$$

where $\bar{F}(n, k)$ is the average value of $F_{n}(k)$ over a 30-s window centered on frame $k$. To ensure that $\bar{F}(n, k)$ captures the baseline background in the presence of sparse pulsatile activity, the computation of $\bar{F}(n, k)$ was as in Sood et al. (2019) and excluded values of $F_{n}(k)$ in the window above the 80th percentile. ROls were numbered consecutively along the anteroposterior direction.

\section{Time-frequency analysis}

A continuous wavelet transform was applied to every $\Delta F_{n} / F$ time series (analytic Morlet wavelet, 10 voices per octave), and the resultant scalograms $\mathcal{S}_{n}(f, k)$ were used to detect transient and sustained oscillations in the ROls. First, for any ROI $n$, the combinations $(f, k)$ of frequency $f$ and frame $k$ at which the magnitude $\left|\mathcal{S}_{n}(f, k)\right|$ was above a threshold $T_{1}$ identified transient oscillations (i.e., occurring at frame $k$ ) with period $1 / f$ in the ROI. Second, for any ROI $n$, the cumulative magnitude $\overline{\mathcal{S}}_{n}(f)=\sum_{k}\left|\mathcal{S}_{n}(f, k)\right|$ was computed at all frequencies $f$, and the values $f^{*}$ at which $\overline{\mathcal{S}}_{n}\left(f^{*}\right)$ was maximum and exceeded a threshold $T_{2}$ identified the presence of sustained activity (i.e., throughout the entire time series) at frequencies $f^{*}$ in the ROI. Threshold $T_{1}$ was set as the 95th percentile of the values $\left|\mathcal{S}_{n}(f, k)\right|$ computed across all frequencies $f$, frames $k$, and ROls $n$, and aimed to isolate the most prominent transient oscillations throughout the acute slice. Threshold $T_{2}$ was set as the 95th percentile of the values $\overline{\mathcal{S}}_{n}(f)$ across all frequencies $f$ and ROls $n$. The combination of conditions (1) $\overline{\mathcal{S}}_{n}\left(f^{*}\right) \geq \overline{\mathcal{S}}_{n}(f)$ for all $f$ and (2) $\overline{\mathcal{S}} n\left(f^{*}\right)>T_{2}$ guaranteed that only the most prominent sustained oscillations throughout the slice were retained.

Finally, the cumulative spectral content of every time series $\Delta F_{n} / F$ was computed in two separate frequency bands, i.e., $0.02-0.2 \mathrm{~Hz}$ (LF band) and $0.2-2.0 \mathrm{~Hz}$ (highfrequency band; HF). The spectral content, $\mathcal{P}_{B}(n)$, of the series $\Delta F_{n} / F$ in the frequency band $B(B=L F, H F)$ was 
computed as the integral of $\left|\mathcal{S}_{n}(f, k)\right|$ over $B$ and the number of frames, i.e.,

$$
\mathcal{P}_{B}(n)=\frac{1}{F_{s}} \sum_{k} \int_{B}\left|\mathcal{S}_{n}(f, k)\right| d f .
$$

\section{$\Delta F / F$ calculations for pulse detection and measurements of amplitude and duration}

ROls exhibiting a pulsatile behavior were identified as follows. First, for any ROI $n$, the normalized series $\Delta F_{n} / F$ was evaluated for skewness (kurtosis value $K \geq 4$ ). If a series met the criteria for skewness, candidate peaks were selected by isolating local maxima with a minimum interpeak distance of 30 frames. Then, for each candidate peak, three measures were computed, i.e., peak prominence, peak width $w$, and root mean square (RMS) in the interval of length $2 w$ centered on the peak. Candidate peaks were rejected if the prominence was lower than 3.5 or the RMS was lower than 2. Threshold values on prominence, RMS, and interpeak distance were determined offline based on the morphology and signal-to-noise ratio of the pulses in a sample series $\Delta F_{n} / F$. Finally, an ROI captured pulsatile activity if the corresponding time series $\Delta F_{n} / F$ retained three or more peaks after rejection. For $\Delta \mathrm{F} / \mathrm{F}$ measurements of amplitude, duration, and events/s, we used image files containing all 6000 frames imported to Fiji-ImageJ (https://imagej.net/Fiji; RRID:SCR_002285). To determine the $\Delta \mathrm{F} / \mathrm{F}$ for the anatomic regions representing the CA3 area of the hippocampus or the posterior, medial, or anterior cortex, we measured the fluorescence values by drawing a shell around these areas. Fluorescence data were then extracted using the image-stack-plot $z$ built-in Fiji function. The data were then imported to AxoGraph ver. 1.7 .5 (https://axograph.com/; RRID:SCR_014284) for automated event detection. The fluorescence changes $(\Delta \mathrm{F} / \mathrm{F})$ were calculated by subtracting each data point from the mean of the baseline fluorescence values. $\Delta \mathrm{F} / \mathrm{F}$ amplitude (calcium events with amplitude $\Delta F / F>0.01$ were identified as events) and duration (50\% of the event peak width) were analyzed using detection algorithms implemented in AxoGraph.

\section{Determination of wave propagation velocity}

To assess the propagation of oscillations across the different ROls, we tracked the migration of calcium waves through the fluorescence time series in each hemisphere. Specifically, for every time series $\Delta F_{n} / F$, we computed the envelope (i.e., absolute value of the analytic signal obtained via Hilbert transform) and retrieved the envelope's highest peak, $p_{n}^{*}$. Then, we retained only those ROls that (1) had a peak $p_{n}^{*}$ with prominence $>3.5$ and (2) presented a sustained oscillation, i.e., an oscillation with frequency $f^{*}$ as defined above, see Time-frequency analysis. Finally, the retained ROls were sorted according to the arrival time, $t_{n}^{*}$, of $p_{n}^{*}$, and the propagation velocity was estimated for consecutive ROls. Namely, denoted with $i_{1}, i_{2}, i_{3}, \ldots$, the ROls after sorting, we measured the propagation velocity between ROls $i_{1}$ and $i_{2}$, ROIs $i_{2}$ and $i_{3}$, etc., according to the formula:

$$
v_{j}=\frac{\left\|C_{i_{j}}-C_{i_{j+1}}\right\|}{t_{i_{j+1}^{*}}^{*}-t_{i_{j}}^{*}},
$$

where $v_{j}$ denotes the velocity in propagating from $\mathrm{ROI} i_{j}$ $(j=1,2,3, \ldots)$ to the next, $C_{i_{j}}$ is the centroid of the ROI $i_{j}$, and $\|\cdot\|$ denotes the Euclidian distance.

For each slice, the propagation velocity was reported as the average $v_{j}$ (mean $\pm \mathrm{SD}$ ) across the pairs of consecutive ROIs. If $<7$ values $v_{j}$ (i.e., eight ROIs) contributed to the propagation velocity, the slice was deemed not showing oscillatory activity, and no propagation velocity was computed. Also, if two or more ROls had their peak $p_{n}^{*}$ arriving at the same time $t_{n}^{*}$, it was concluded that the ROls were part of the same wavefront. Accordingly, the ROls were preemptively combined into one region, and the average distance between the centroids of these ROls and the centroid of the next ROI in the sequence was used in formula (3). The use of the Hilbert-transform and the signal envelope aimed to reduce the impact of noise on the time series and better capture the peak of the normalized fluorescence intensity, which was used as a proxy for a traveling wavefront. Also, the ROI aggregation and sorting procedures described above aimed to capture the spatial migration of a traveling wave.

\section{Local field potential (LFP) recordings}

LFP recordings were taken in parallel with the imaging sessions. LFPs were recorded using an RHD2000 multichannel amplifier (Intan Technologies) acquired with RHD2000 interface software (version 1.5, copyright Intan Technologies). LFP recordings were acquired at $20 \mathrm{kHz}$, low-pass filtered (eighth order Chebyshev Type I IIR filter with cutoff frequency at $80 \mathrm{~Hz}$ and zero-phase distortion), and downsampled to $200 \mathrm{~Hz}$.

\section{Statistics}

Statistical analyses were performed using GraphPad Prism 8 software (GraphPad; RRID:SCR_002798). Summary data are expressed as bar graphs, box plots, or violin plots. Box plots show the interquartile range extending from the 25th and 75th percentiles, whereas the box plot whiskers illustrate the minimum and maximum values. We used box plots to summarize the distribution of the results and to allow the reader to quickly compare data between conditions. Violin plots allow better visualization of the distribution of the data points than box plots; thus, we used violin plots to demonstrate the full effect of the loss of Kcnq2 or Kcnq3 on the amplitude, duration, and events/s of the calcium responses. For analysis, we used unpaired or paired Student's $t$ tests, MannWhitney $U$ non-parametric unpaired tests, or Wilcoxon paired tests. Non-parametric tests were used for datasets in which the distribution of the data failed normality tests.

\section{Results}

KCNQ2 channels are expressed in neonatal excitatory neurons; however, it is currently unknown how KCNQ2 


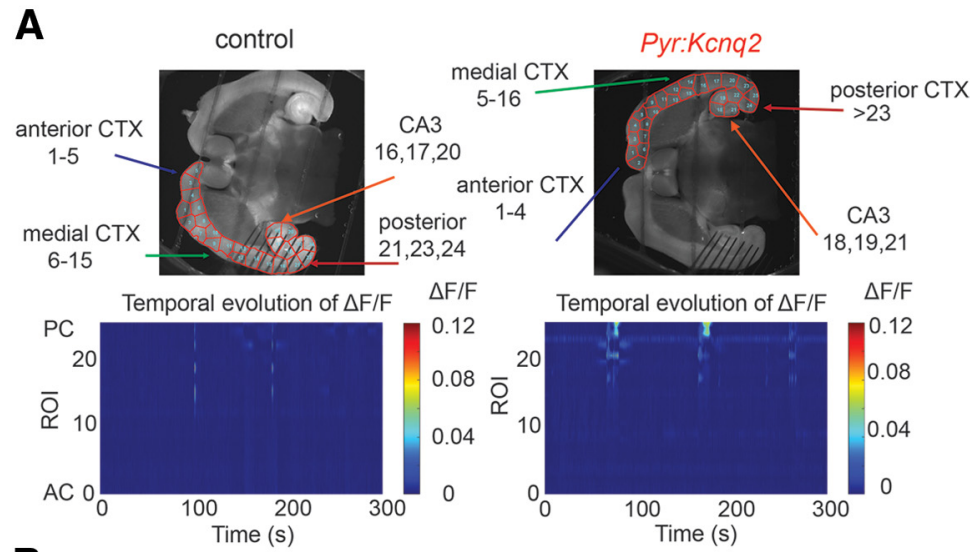

B control
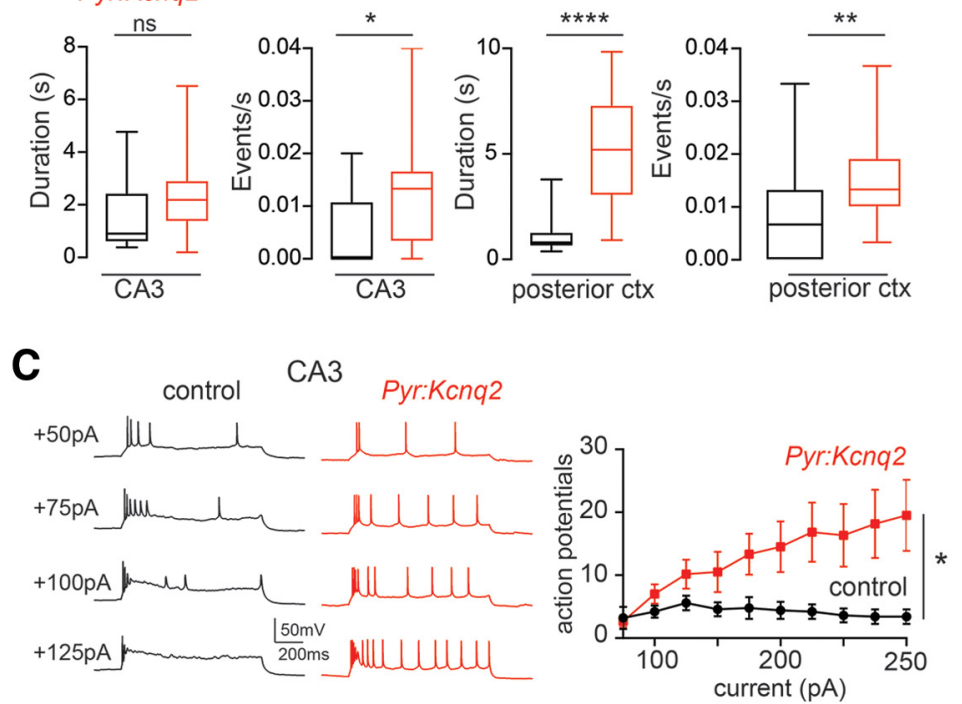

Figure 1. Ablation of Kcnq2 from neonatal excitatory neurons leads to increased excitability in the hippocampus and posterior cortex. All recordings were in the presence of $2.5 \mathrm{~mm}$ Ko. A, top panels, Examples of acute slices from control and Pyr:Kcnq2 mice with one hemisphere segmented into ROls. Bottom panels, Representative $2 \mathrm{D}$ plots showing calcium activity $(\Delta \mathrm{F} / \mathrm{F})$ as a function of time for the different ROls. The numbering corresponds to the segmented area shown on the top panels with lower values toward the anterior cortex $(\mathrm{AC})$ and higher values toward the CA3 area of the hippocampus and posterior cortex (PC). $\boldsymbol{B}$, Summary graphs show the effect of Kcnq2 ablation on the calcium event duration and frequency for the CA3 area of the hippocampus and the posterior cortex. Data in the box plots show the median and the interquartile range. $\left({ }^{*} p<0.05,{ }^{* *} p<0.01,{ }^{* * * *} p<0.0001\right)$. C, Whole-cell recordings from CA3 pyramidal neurons show that ablation of Kcnq2 increases neuronal excitability. Left panels, Family of depolarizing 1-s current steps in either control or Pyr:Kcnq2 slices. Right, Summary graph shows the number of recorded action potentials in control and Pyr:Kcnq2 CA3 pyramidal neurons. ${ }^{*} p<0.05$ compared with control group. For $\boldsymbol{C}$, data are represented as mean \pm SEM. Additional details on the statistical analysis for this figure are found in Table 1 under the Figure 1 section.

dysfunction alters forebrain network excitability early in development. To address this question, we investigated the role of KCNQ2 channels in controlling excitatory neural activity in developing neurons using Kcnq2 conditional knock-out mice. To define the spatial and temporal pattern of excitatory neurons in acute slices and across the neocortex, we generated Emx $1^{\text {cre }}:: P C$-G5-tdT::Kcnq2 $2^{f / f}$ mice, denoted hereafter as Pyr:Kcnq2 mice. In the presence of Cre recombinase, the Pyr:Kcnq2 mice express the genetically encoded calcium sensor GCamp5g (Gee et al., 2014), which allowed us to visualize calcium events. Additionally, these mice express td-Tomato, enabling us to determine the extent of Cre recombinase activity and specificity. To maintain the circuitry between regions, we used horizontal slices from neonatal mice (P4P7). We focused on this developmental age range because it is a critical period for the development and maturation of synaptic connectivity, corresponding to a prenatal period in humans.

\section{Pyr:Kcnq2 neonatal slices exhibit increased excitability}

Figure $1 A$ compares the widefield calcium activity in horizontal slices from control and Pyr:Kcnq2 mice. In slices of both groups, the area covering each hemisphere was decomposed into ROls using the SLIC clustering algorithm, which aggregates pixels in a $\mathrm{ROI}$ if they are spatially 
contiguous and of similar fluorescence intensity. In this way, ROls were constructed as macro-areas that have similar fluorescence intensities across neighbor pixels, which assures anatomic homogeneity within each macro-area. Also, because of the intra-ROI homogeneity, the fluorescence signal was averaged across pixels in each $\mathrm{ROI}$, thus reducing the effects of noise on the analysis of the fluorescence time series.

The results of the ROI partition indicate that Pyr:Kcnq2 slices and control slices had a similar number of ROls (31 \pm 0.5 ROls, $n=52$ hemispheres vs $31 \pm 0.5$ ROls, $n=64$ hemispheres; Pyr:Kcnq2 vs control $p=0.62$, Student's $t$ test; $t=0.4971$, $\mathrm{df}=114$ ) and ROls of similar size (number of pixels per ROI: $971 \pm 12$ pixels, $n=52$ hemispheres vs $963 \pm 9$ pixels, $n=64$ hemispheres; Pyr: Kcnq2 vs control $p=0.91, t=0.4971, \mathrm{df}=114$ Student's $t$ test). Thus, we observed no significant anatomic or functional differences between the two groups of slices.

Previous work has shown that spontaneous neuronal activity is infrequent and primarily confined to the hippocampal formation and entorhinal cortex in neonatal slices (Garaschuk et al., 2000; Namiki et al., 2013). Indeed, during a 5-min recording from control littermate slices, we observed calcium activity in the CA3 region of the hippocampus and the posterior cortex, which primarily represented the entorhinal cortex (Fig. 1A, bottom panels). As observed in previous studies, the activity was modest in intensity, with brief pulsatile periods and few events/s (Fig. 1B).

Next, we examined slices from mice lacking Kcnq2 in excitatory cells. We note that the Emx1-cre mice express Cre recombinase as early as E10.5 (Gorski et al., 2002), thus, by P4, KCNQ2 levels in the majority of excitatory cells would be minimal. Consistent with this assertion, we found CA3 pyramidal neurons from Pyr:Kcnq2 mice fired a greater number of action potentials following depolarizing current injections (Fig. 1C). Furthermore, in the absence of Kcnq2, calcium responses remained confined to the same pacemaker regions as in slices from control littermate mice, i.e., the CA3 region of the hippocampus and the posterior cortex (Fig. 1A). However, because of the loss of Kcnq2, the number of events during the recording period was significantly higher in both regions (0.012-0.015 events/s; approximately four to five events in 5 min; Fig. 1B). In addition to identifying changes in the number of detected events, we found that the duration but not amplitude of the calcium events in the posterior cortex was greater in the knock-out mice (control: $0.073 \pm 0.014 \Delta \mathrm{F} / \mathrm{F}, \quad n=14$ hemispheres; Pyr:Kcnq2: $0.016 \pm 0.03 \Delta \mathrm{F} / \mathrm{F}, n=20$ hemispheres; $p=0.08$ MannWhitney $U$ test; Fig. 1B). By contrast, we observed no changes in the amplitude or duration of the calcium signal for the CA3 region of the hippocampus (control: $0.031 \pm$ $0.006 \Delta \mathrm{F} / \mathrm{F}, n=9$ hemispheres; Pyr:Kcnq2: $0.034 \pm 0.005$ $\Delta \mathrm{F} / \mathrm{F}, n=18$ hemispheres; $p=0.97$ Mann-Whitney $U$ test; Fig. $1 B$ ). The observed differences between the CA3 region of the hippocampus and the posterior cortex suggested that deletion of Kcnq2 manifests in a distinct manner in different macro-circuits.

As with the slices from control animals, we did not detect any consistent large calcium transients during the 5- min recording window in the neocortex. Similarly, earlier work showed that overall cortical activity in acute slices is very low at this developmental time point (Garaschuk et al., 2000). We note that our fluorescence signal represented calcium activity across all neuronal subcompartments; thus, at this resolution, we could not determine whether the changes in calcium signals were because of increased excitability at dendrites, soma, or axons. These changes were most likely because of a combination of all three subcompartments, as KCNQ2 channels are not simply restricted to axons in the early developmental period.

To further probe the effects of Kcnq2 deletion from the neocortex under more excitable conditions, we imaged slices that had been incubated with $8 \mathrm{~mm}$ extracellular potassium (Ko) for $\sim 20 \mathrm{~min}$. The use of $8 \mathrm{~mm}$ Ko allowed us to compare the activity of different regions under constant stimulation conditions. A concentration of $8 \mathrm{~mm}$ Ko was necessary to increase the event number in our slices (Fig. 2). We applied $8 \mathrm{~mm}$ Ko to slices for no more than $20 \mathrm{~min}$ to prevent the slices from becoming epileptic (i.e., from exhibiting spontaneous ictal events following removal of the high-potassium solution). After incubating the slices with $8 \mathrm{~mm} \mathrm{Ko}$, we recorded 5-min time-lapse movies. To complement our calcium imaging, we also recorded LFPs from the CA3 region of the hippocampus, which allowed us to correlate calcium activity to the overall network activity. Similar to the recordings in low-potassium artificial cerebrospinal fluid, we found that the majority of calcium events in slices from control animals originated from the hippocampal formation and posterior cortex, closely following the measured LFPs (Fig. 2B; Movie 1).

In contrast to the control slices, slices from mice lacking KCNQ2 channels exhibited widespread calcium activity spanning all cortical regions and layers (Fig. 2; Movie 2). Closer examination of the time-lapse movies also showed that activity in the neocortex frequently initiated in the upper cortical layers and then migrated to the deeper layers and neighboring neocortical regions (see Movie 2). Calcium waves propagated in multiple directions and were generally multifocal, as the initial activity sprang at multiple regions. We also found that the calcium events were larger and longer lasting across all regions (Fig. 2C). Additionally, the average propagation velocity of the calcium responses across ROls in slices from Pyr:Kcnq2 mice was $0.64 \pm 0.074 \mathrm{~mm} / \mathrm{s}$ ( $n=55$ hemispheres), which was almost three times slower than the rate measured in control slices $(2.07 \pm 0.53 \mathrm{~mm} / \mathrm{s}, n=18$ hemispheres, $p=0.0006$ Mann-Whitney $U$ test). We currently do not know the reason for this difference, but there could be many contributing factors such as the location of the calcium signal, or the presence of multiple waves with different latencies instead of one large, synchronous wave. We did not investigate this further.

Although the calcium activity was robust in CA3, we noticed that the LFPs were typically smaller or absent in slices from Pyr:Kcnq2 mice. This result could be attributed to several reasons, such as electrode placement, increased GABAergic receptor activity (see below), the higher tendency of Kcnq2-null excitatory neurons to show spreading depression and persistent depolarization leading to sodium 

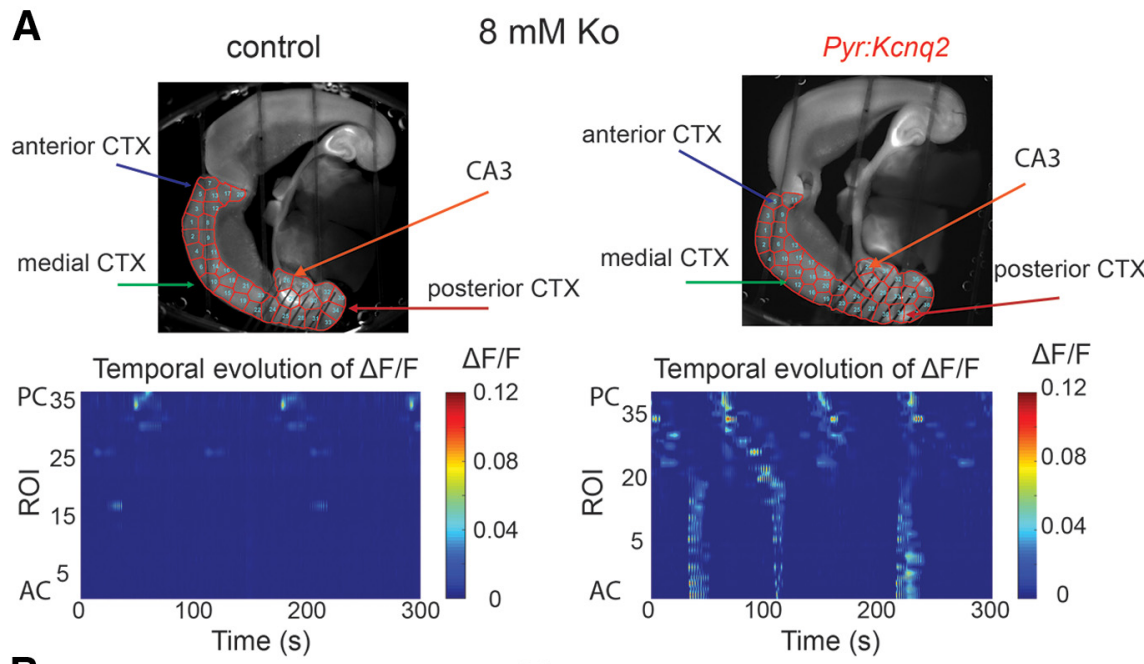

B

CA3
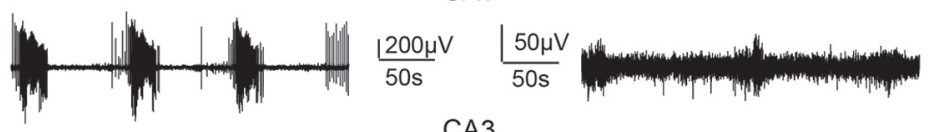

CA3
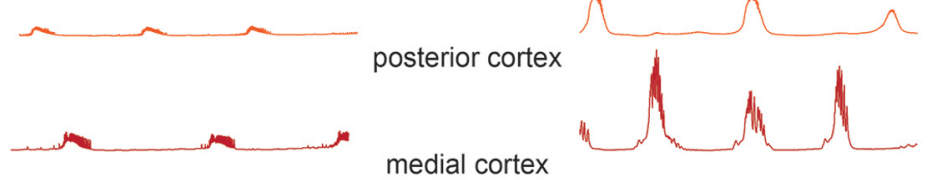

medial cortex
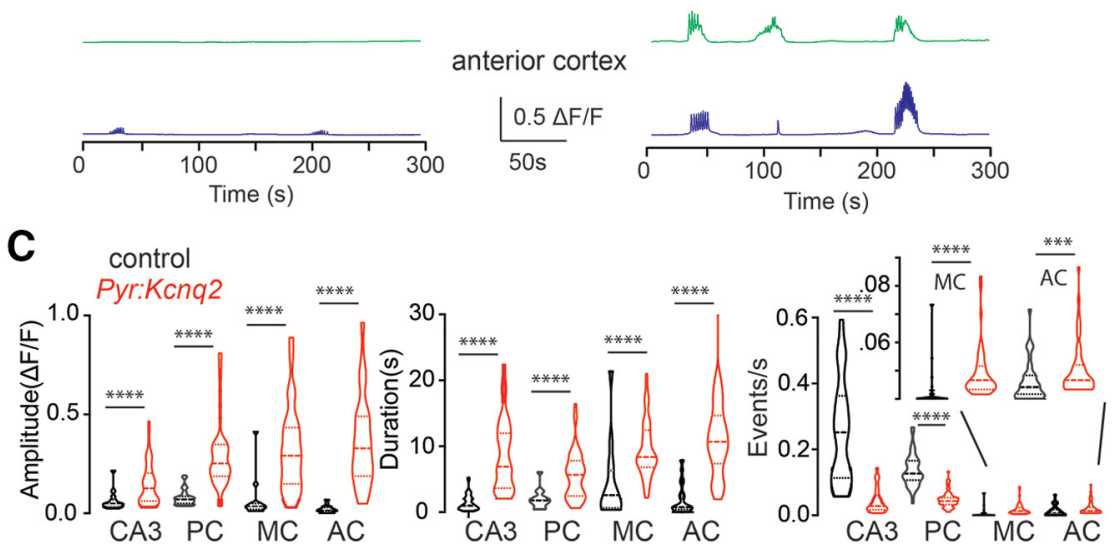

Figure 2. Deletion of Kcnq2 from excitatory neurons leads to elevated calcium activity across the forebrain in $8 \mathrm{~mm}$ Ko. $\boldsymbol{A}$, top panels, Examples of acute slices from control and Pyr:Kcnq2 mice with one hemisphere segmented into ROls. Bottom panels, 2D plots show the calcium activity across the different ROls. The numbering corresponds to the segmented area shown on the top panels with lower values toward the $\mathrm{AC}$ and higher values toward the PC. Note that in the absence of $K c n q 2$, substantial calcium activity is measured across all regions of the forebrain. $\boldsymbol{B}$, top two panels, Temporal evolution of the LFPs and $\Delta \mathrm{F} / \mathrm{F}$ recorded in parallel in the CA3 region of the hippocampus. Note that in contrast to the LFPs in slices from Pyr:Kcnq2 mice, the calcium responses are large and long lasting. Middle and bottom panels, Temporal evolution of the $\Delta F / F$ across multiple ROls. $\boldsymbol{C}$, Violin plots show the effect of $K c n q 2$ deletion on the amplitude, duration, and frequency of the calcium events for different anatomic regions. MC refers to the medial cortex. Note that ablation of Kcnq2 led to a large and uniform increase of the calcium response amplitude and duration. ${ }^{\star * \star *} p<0.0001$. Additional details on the statistical analysis and number of replicates for this figure are found in Table 1 under the Figure 2 section.

channel inactivation (Aiba and Noebels, 2021), or a combination of these mechanisms. We did not pursue this question further. Importantly, independent of the region being imaged, we observed an increase in both the amplitude and duration of calcium events (Fig. 2C). This trend was particularly striking in the medial and anterior cortex, where the event amplitude increased by $\sim 50$ fold (i.e., medial cortex: from $0.02 \Delta F / F$ to $0.33 \Delta F / F$ ).
In slices from Pyr:Kcnq2 mice, large calcium events in CA3 and the posterior cortex were organized in LF patterns. Additionally, the average number of events in the calcium fluorescence signals in both regions was decreased compared with the number of events from control slices (CA3, decreased from 0.025 to 0.004 events/s; posterior cortex, decreased from 0.013 to 0.005 events/s; Fig. $2 C$ ). This shift in the number of events reflects the 


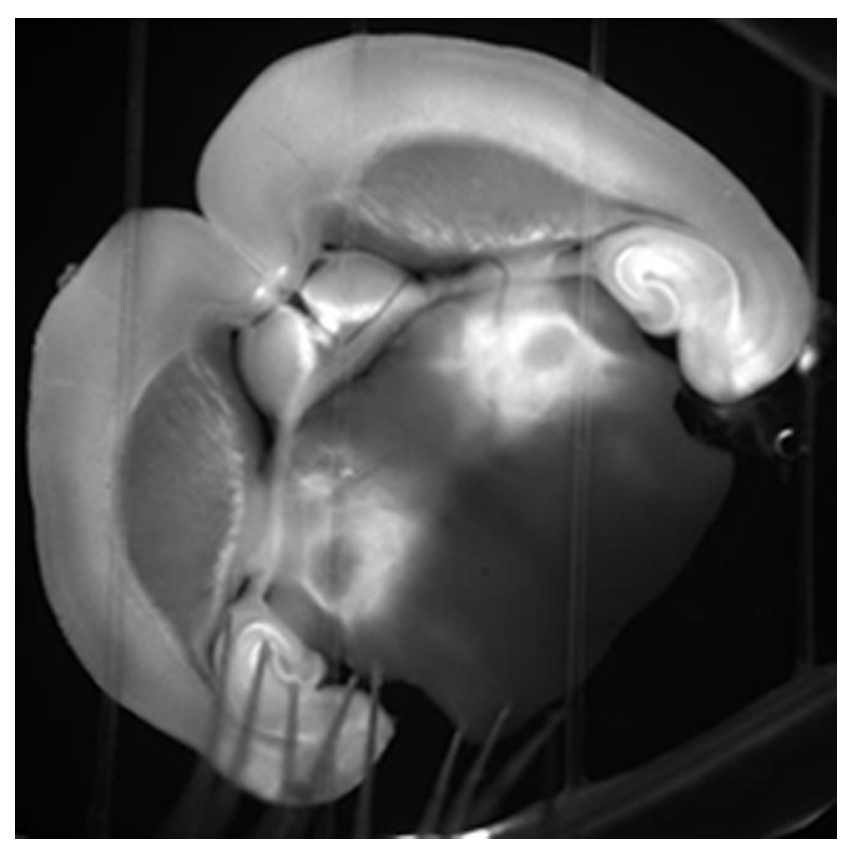

Movie 1. Representative imaging showing the forebrain calcium activity in control slices in the presence of $8 \mathrm{mM} \mathrm{Ko}$. Note the activity in the hippocampal formation. Movie played at $5 \times$ speed. [View online]

loss of small events and the emergence of longer-lasting less frequent large population events. Indeed, the duration of average calcium events increased from 1.4 to 8.5 and 2.2 to $6.5 \mathrm{~s}$ in the CA3 region of the hippocampus and the posterior cortex, respectively. In contrast, in the medial and anterior cortex, we primarily observed an increase rather than a decrease in the number of calcium events (medial cortex: 0.006 to 0.018 events/s; anterior cortex: 0.012 to 0.021 events/s; Fig. $2 C$ ), which primarily reflects the appearance of prolonged synchronous activity following the large depolarization of the posterior cortex and the CA3 region of the hippocampus. Thus, based on these data, we conclude that the loss of Kcnq2 from excitatory neurons increases excitability, manifested as large population events, across the forebrain, independent of the region. This trend is consistent with the wide distribution of KCNQ2 channels in the forebrain (Cooper et al., 2001).

\section{Kcnq2 deletion leads to transient slow-wave patterns}

To further quantify the effects of Kcnq2 deletion on the calcium signals from excitatory cells, we conducted a time-frequency analysis of the fluorescence time series (Fig. 3) and measured the cumulative magnitude of these signals in the LF $(0.02-0.2 \mathrm{~Hz})$ and HF $(0.2-2 \mathrm{~Hz})$ bands, which captured the majority of the activity in our slices. Figure $3 A$ shows the time-frequency analysis averaged across all ROIs and indicates that the loss of Kcnq2 primarily increased activity in the LF band. Moreover, Figure $3 B$, left panels, shows the sample distribution of the frequency $f$ of the transient oscillations across all ROls for

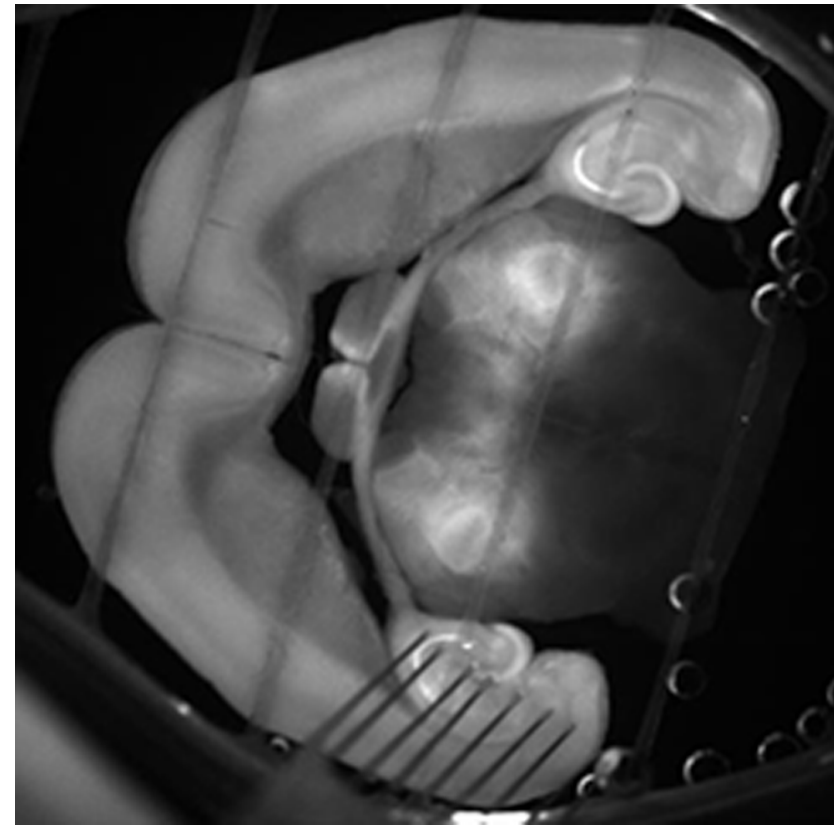

Movie 2. Representative imaging showing the forebrain calcium activity in Pyr:Kcnq2 slices in the presence of $8 \mathrm{~mm}$ Ko. Note the migrating activity across the neocortex. Movie played at $5 \times$ speed. [View online]

Kcnq2 (red line) and control (black line), respectively, whereas Figure $3 B$, right panels, reports the number of ROls that exhibited sustained oscillations (i.e., a histogram of frequencies $f^{*}$ ). All panels refer to the slices in Figure $2 A$. Figure $3 A, B$ shows that deleting $K c n q 2$ is associated with widespread oscillations at frequencies concentrated in the LF band. This result was confirmed at the population-level, i.e., we reported that, across all slices, the oscillations spanned a larger number of ROls in Pyr: Kcnq2 slices than control slices (Fig. $3 C, D$ ), were sustained throughout the duration of the recording period, and exhibited transient modulations of the fundamental frequency, although the fundamental frequency remained primarily confined to the LF band (Fig. $3 C, D$ ). In contrast, few ROls showed significant periodic oscillations in the $\mathrm{HF}$ range, as summarized in Figure $3 D$.

Together, these findings are consistent with our earlier observation that loss of Kcnq2 primarily leads to the appearance of large but less frequent population events (see Fig. 2). However, these data also show that deletion of Kcnq2 from forebrain excitatory neurons leads to multiple hot spots at low frequencies in the presence of elevated extracellular potassium. These hot spots are distributed across the entire slice and can be spatially separated but retain similar frequencies, which strongly indicates that the hot spots could stem from increased network signaling.

\section{GABAA receptor activity amplifies without shaping Kcnq2 slow-wave patterns}

To further understand the role of network signaling in the formation of LF hot spots, we conducted follow-up 

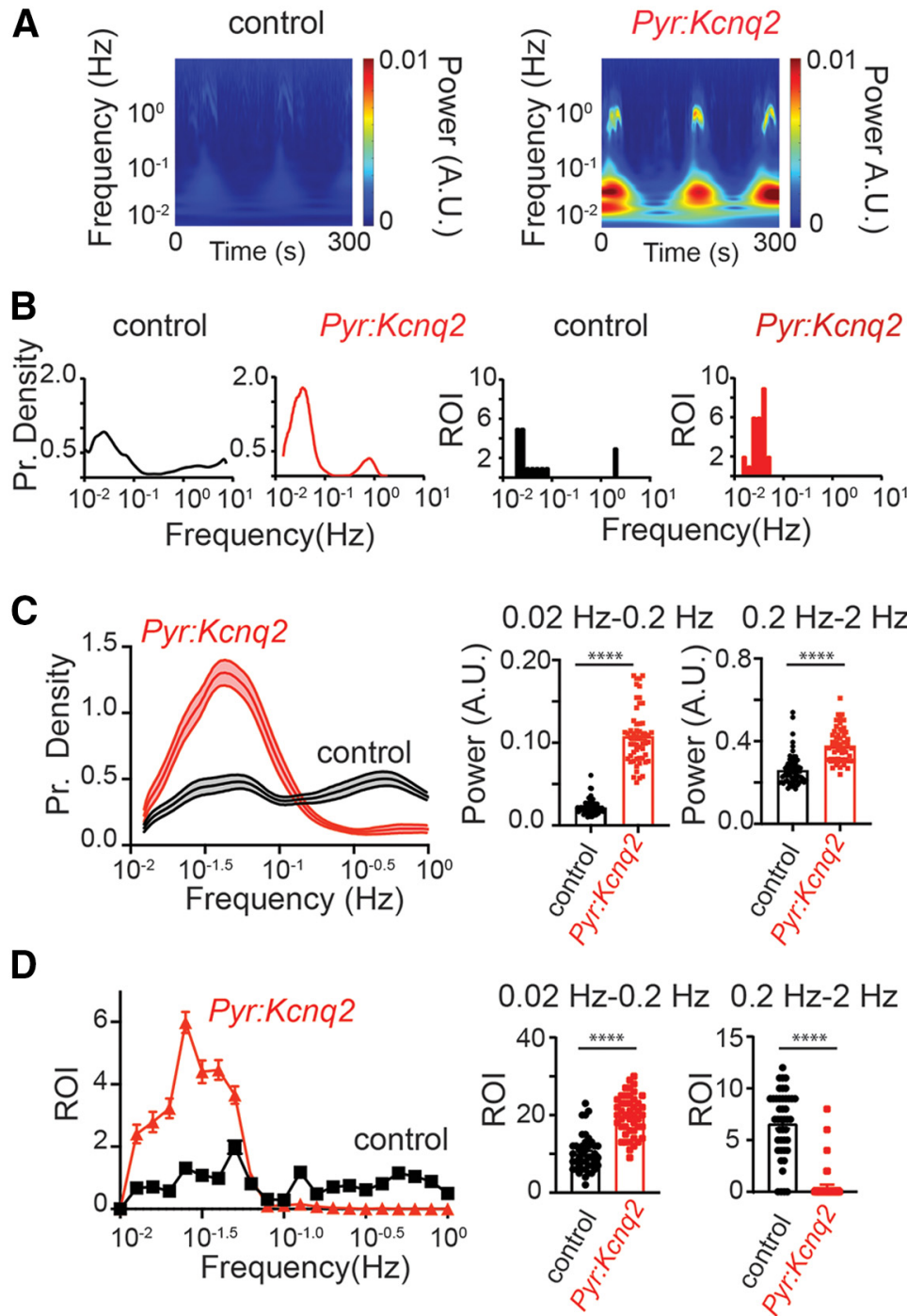

Figure 3. Deletion of Kcnq2 leads to LF calcium oscillations. Deletion of Kcnq2 increased the likelihood of LF oscillations across a large number of ROls; oscillations were sustained throughout the duration of the recording period. All recordings were in the presence of $8 \mathrm{~mm}$ Ko. A, Wavelets from control and Pyr:Kcnq2 hemispheres. The wavelets were generated from the examples shown in Figure $2 A$. Note the large increase in the power for the oscillations occurring at the LF range in the hemisphere from Pyr:Kcnq2 animals. Although in this example some increases in the power were also observed at the higher-frequency values, this was not seen across all slices, as shown in panel $\boldsymbol{C}$, right panels. $\boldsymbol{B}$, Comparison of the probability density of the frequency $f$ of transient oscillations (left panels) and the number of ROls undergoing sustained oscillations (right panels) for the examples depicted in panel $\boldsymbol{A}$. $\boldsymbol{C}$, left, Comparison of the probability density of the transient oscillations for control $(n=43)$ and Pyr:Kcnq2 hemispheres $(n=47)$ across multiple slices. Data are represented as mean \pm SEM. Middle and right panels, Summary graphs quantifying the power measured for the two frequency domains, $0.02-0.2$ and $0.2-2 \mathrm{~Hz}$. Note that loss of Kcnq2 increased the likelihood of observing LF calcium oscillations ranging primarily from 0.03 to $0.05 \mathrm{~Hz}$. Data are represented as mean $\pm \mathrm{SEM}\left({ }^{* * *} p<0.0001\right)$. $\boldsymbol{D}$, left, Comparison of the ROls undergoing sustained oscillations for control $(n=43)$ and Pyr:Kcnq2 $(n=47)$ hemispheres. Results are reported for sustained oscillations whose frequency $f^{*}$ is in the LF and the HF range, respectively. See Materials and Methods, Time-frequency analysis, for a definition of the frequency $f^{*}$ of a sustained oscillation. Middle and right panels, Summary graphs of the ROls at two frequency domains, $0.02-0.2$ and $0.2-2 \mathrm{~Hz}\left({ }^{* * *} p<0.0001\right)$. Note that deletion of Kcnq2 leads to a greater number of forebrain regions that show sustained LF calcium oscillations (from 0.012 to $0.05 \mathrm{~Hz}$ ). This is in contrast to control slices that exhibit oscillation frequencies across a wider range of frequencies (from 0.012 to $1.2 \mathrm{~Hz}$ ). Additional details on the statistical analysis and number of replicates for panels $\boldsymbol{C}, \boldsymbol{D}$ are found in Table 1 under the Figure 3 section.

experiments to determine whether these slow oscillations are driven by glutamatergic or GABAergic activity. Figure 4 shows that inhibition of GABAA receptors using $50 \mu \mathrm{m}$ picrotoxin (PTX), a ubiquitous GABAA receptor open channel antagonist that blocks both synaptic and extra-synaptic
GABAA receptors, further exacerbated the increase in network activity in both control and Pyr:Kcnq2 slices (Fig. 4A,B). Picrotoxin also sped up, almost doubling, the propagation velocity of the calcium activity in Pyr:Kcnq2 slices (from $0.59 \pm 0.11$ to $1.08 \pm 0.15 \mathrm{~mm} / \mathrm{s}, n=20$ hemispheres, 

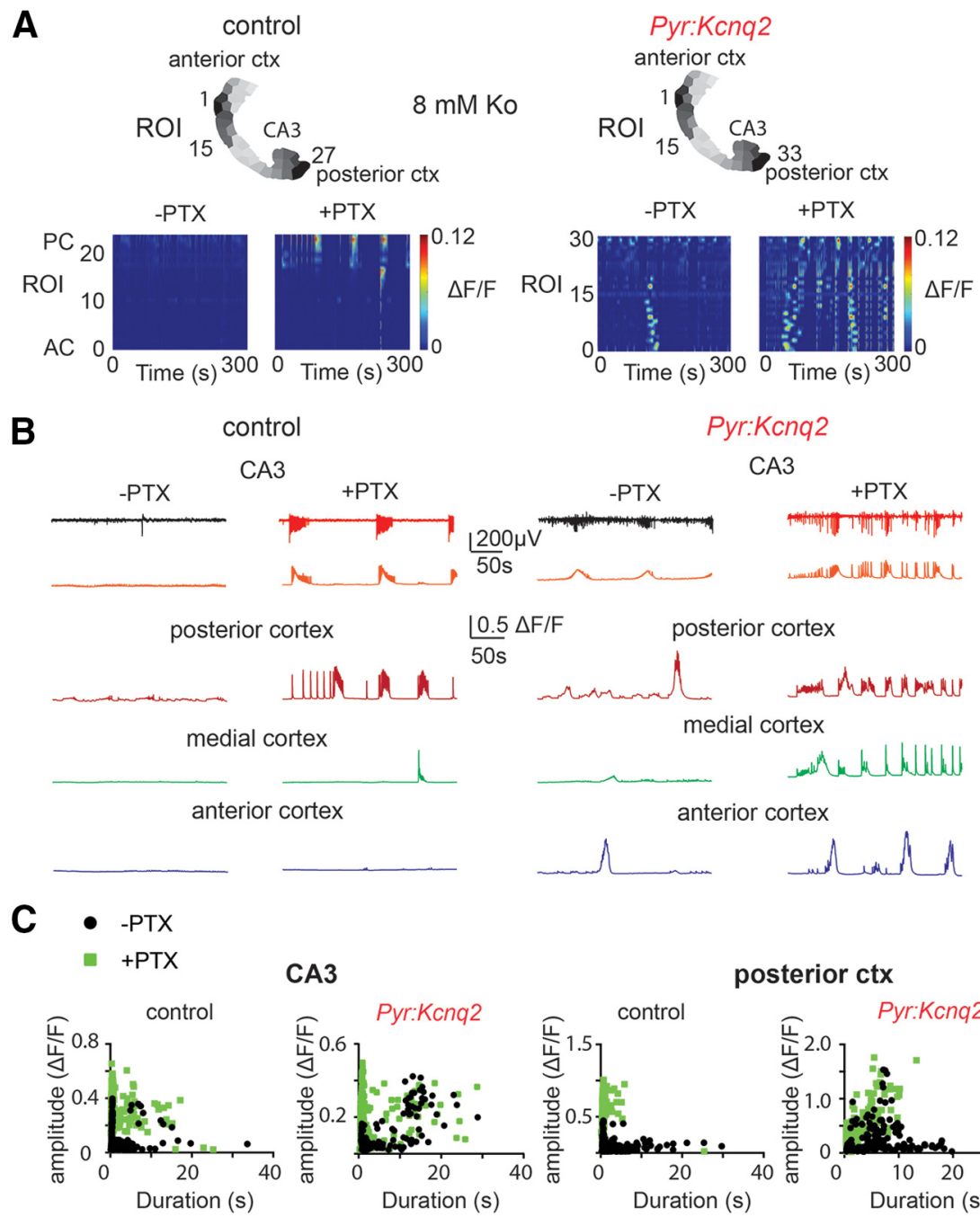

medial CTX
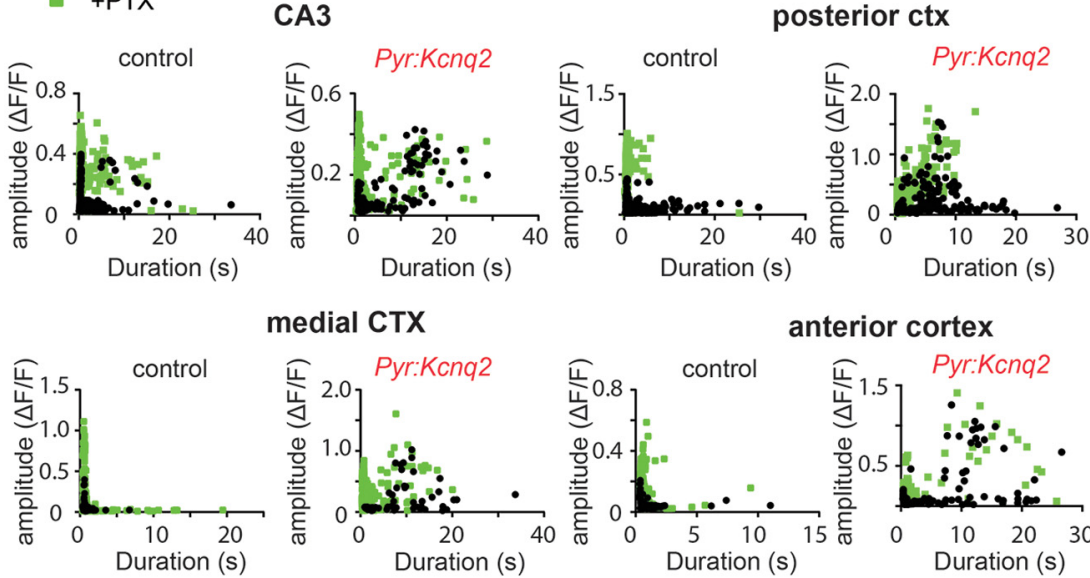

Figure 4. GABAA receptors limit activity in neonatal control and Pyr:Kcnq2 brain slices. All recordings were in the presence of 8 mm Ko. A, Panels show examples of acute slices from control and Pyr:Kcnq2 mice before and after application of $50 \mu \mathrm{m}$ PTX to block GABAA receptors. Top panels, Hemispheres segmented into ROls. Bottom panels, 2D plots representing the changes in the calcium activity $(\Delta F / F)$ across the different ROls on application of PTX. The numbering corresponds to the segmented area shown on top with lower values toward the AC and higher values toward the PC. Note that inhibiting GABAA receptors led to widespread calcium activity in the Pyr:Kcnq2 hemisphere. In contrast, the activity in the control hemisphere was primarily confined to the posterior cortex and the hippocampal formation following application of PTX. B. Calcium responses from the different anatomic regions before and after application of PTX. Note that in Pyr:Kcnq2 slices application of PTX led to the appearance of a barrage of activity across all regions. C, Scatter plots show the effect of PTX on the $\Delta F / F$ amplitude and duration. Note that application of PTX increased the number of larger and faster calcium signals. Additional details on the statistical analysis and number of replicates for this figure are found Table 1 under the Figure 4 section.

$p=0.0136$ Wilcoxon pair test), further suggesting that GABAA receptors limit the spread of calcium responses in the forebrain. Our results are consistent with several studies showing that the application of PTX can increase network activity in the neonatal brain, even when GABA acts as an excitatory transmitter because of its depolarized chloride equilibrium potential in neonatal excitatory neurons (Kirmse et al., 2015; Che et al., 2018), but also see (Murata and Colonnese, 2020). Independent of the genotype, blocking GABAA receptors led to larger fluorescence signal 
A

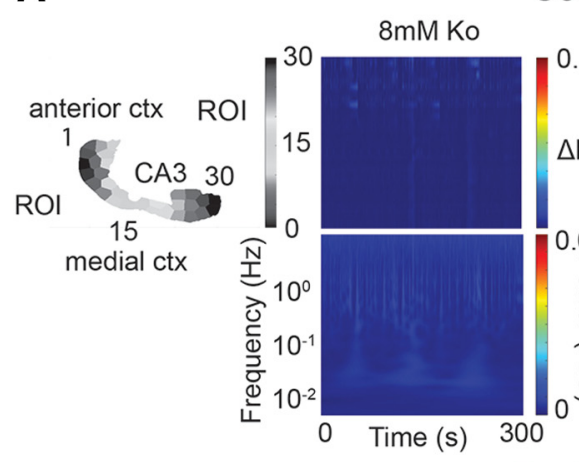

Control

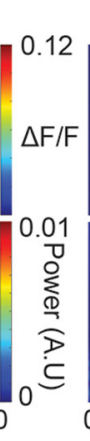

+ PTX

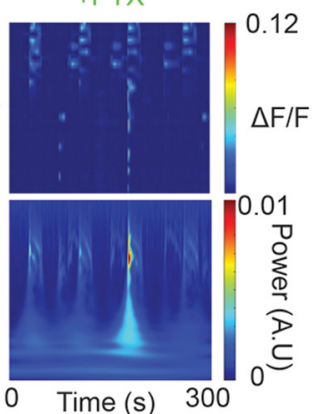

B

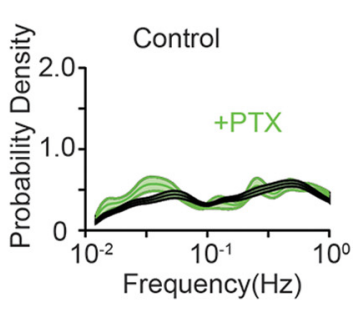

ROI anterior ctx $\mathrm{RO}$
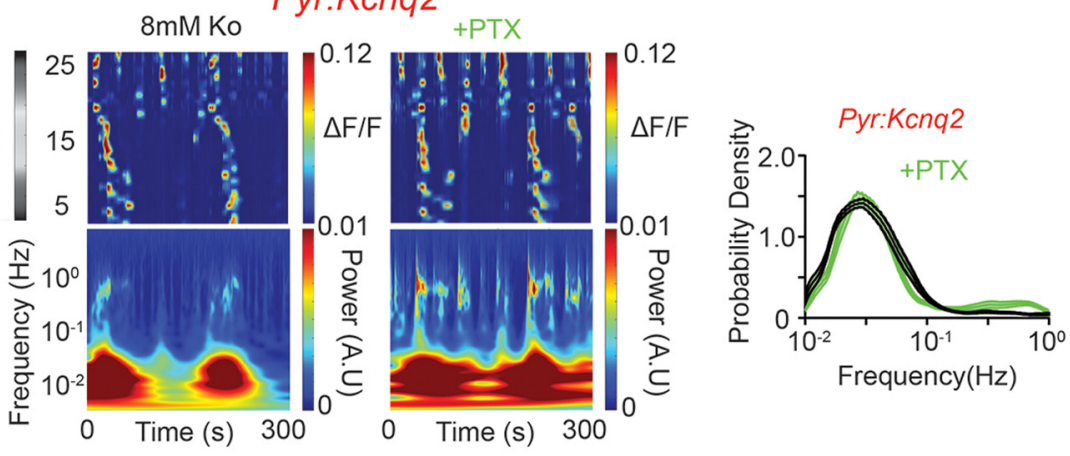

C
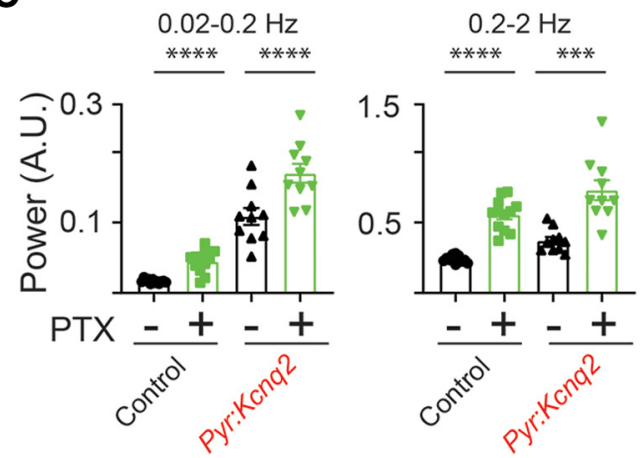

D
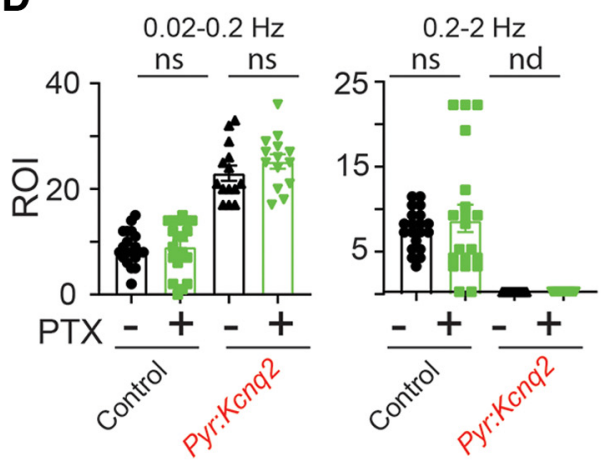

Figure 5. GABAA receptor activity limits the power but not the occurrence of LF calcium oscillations in Pyr:Kcnq2 slices. All recordings were in the presence of $8 \mathrm{~mm}$ Ko. $\boldsymbol{A}$, Representative examples show calcium activity $(\Delta \mathrm{F} / \mathrm{F})$ over time and the corresponding wavelets in the presence and absence of $50 \mu \mathrm{M}$ PTX for control and Pyr:Kcnq2 slices. Note the large increase in power at the LF range in Pyr:Kcnq2 slices, further quantified across multiple slices in panel $\boldsymbol{C}$. $\boldsymbol{B}$, Comparison of the probability density of the frequency $f$ for transient oscillations before and after PTX in control $(n=20)$ and Pyr:Kcnq2 $(n=14)$ hemispheres. Data are represented as mean \pm SEM. The absence of change in the probability density on blocking GABAA receptor activity suggests that GABAA receptors alter the peak oscillation frequency at the different ROls. $\boldsymbol{C}$, Summary graphs show that application of PTX increased the power for the LF and HF domains in control and Pyr:Kcnq2 slices. $\boldsymbol{D}$, Summary graphs show that on application of PTX the number of ROls undergoing sustained oscillations in control and Pyr:Kcnq2 slices did not change. Together, $\boldsymbol{C}, \boldsymbol{D}$ suggest that blocking GABAA receptors primarily increases the activity within each $\mathrm{ROI}$. Data are presented as mean $\pm \mathrm{SEM}\left({ }^{\star \star \star} p<0.001,{ }^{* \star \star \star} p<\right.$ 0.0001). Additional details on the statistical analysis for panels $\boldsymbol{C}, \boldsymbol{D}$ are found in Table 1 under the Figure 5 section. nd, not determined.

amplitudes in all areas of the forebrain (Fig. 4B,C). However, the average calcium event duration decreased as blocking GABAA receptors led to the appearance of larger but briefer calcium signals in the Pyr:Kcnq2 slices (Fig. 4B,C). We also observed this trend in the LFP recordings (Fig. $4 B$ ), suggesting that GABAA receptor activation dampens ongoing network activity in the absence of Kcnq2 across the forebrain. Consistent with uniform upregulation of activity in slices from Pyr:Kcnq2 mice, we found a generalized increment of the time-frequency signal in response to PTX independent of the genotype (Fig. 5A). This resulted in a significant increase of the power both in the LF and HF bands at the populationlevel (i.e., across slices), and the percent increase in power with respect to the pre-PTX case was similar in slices from Pyr:Kcnq2 and control mice. vice versa, the probability density of the frequency $f$ of transient oscillations in a slice (Fig. $5 B, C$ ) and the total number (i.e., across all slices) of ROls exhibiting a sustained oscillation (Fig. 5D) were similar 
A

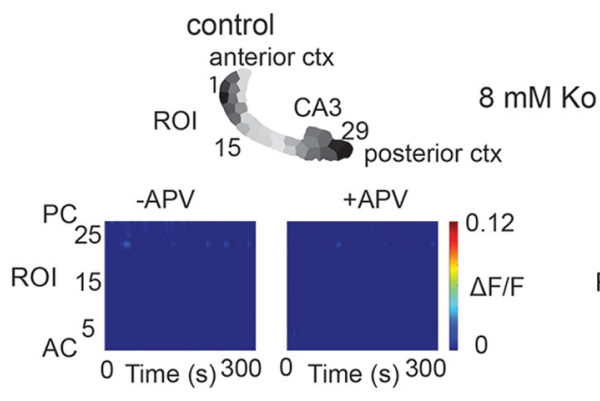

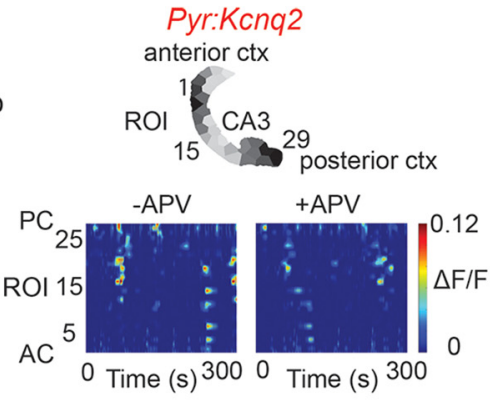

Pyr:Kcnq2
B

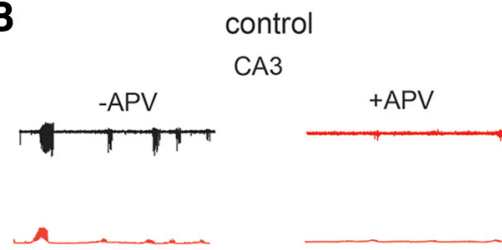

posterior cortex

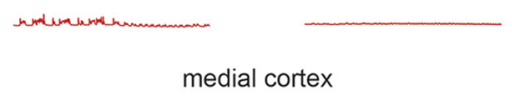

medial cortex
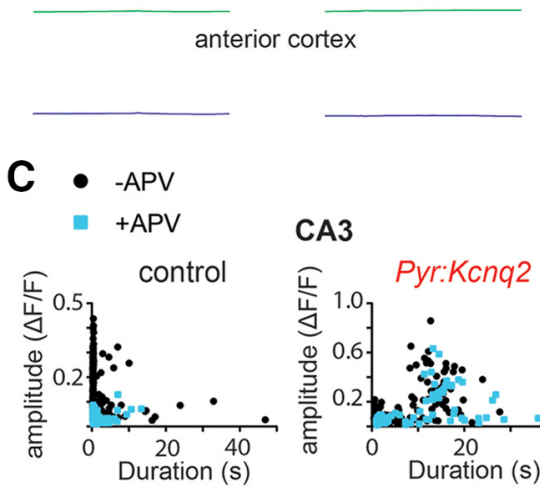

\section{CA3}

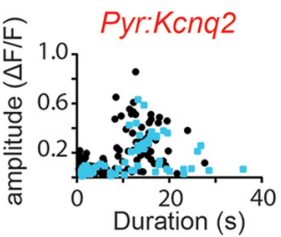

medial CTX
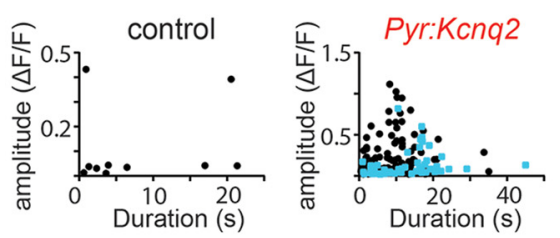
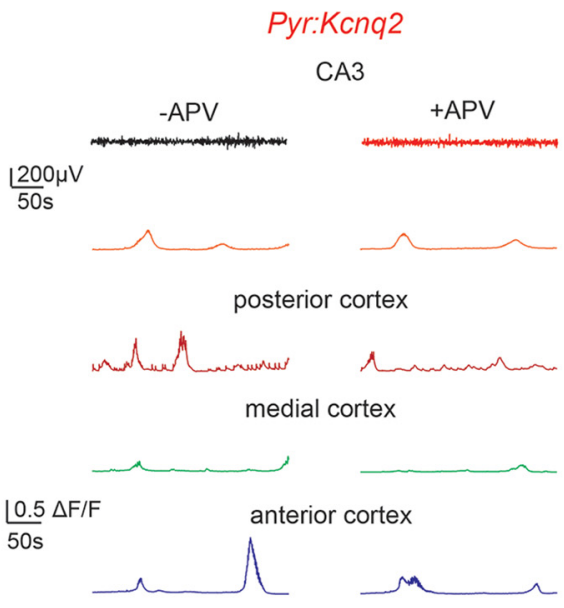

Figure 6. Kcnq2 deficient slices acquired NMDA receptor independent calcium activities. All recordings were in the presence of 8 $\mathrm{mm}$ Ko. A, Panels show examples of acute slices from control and Pyr:Kcnq2 mice before and after application of $25 \mu \mathrm{M}$ D-APV (APV). Top panels, Hemispheres segmented into ROls. Bottom panels, 2D plots representing the $\Delta \mathrm{F} / \mathrm{F}$ as a function of time for the different ROls. The numbering corresponds to the segmented area shown on top with lower values toward the anterior cortex (AC) and higher values toward the posterior cortex $(\mathrm{PC})$. B , Temporal evolution of calcium activity $(\Delta \mathrm{F} / \mathrm{F})$ for the different anatomic regions before and after application of APV. Note that application of APV did not prevent the occurrence of large slow calcium events. $\boldsymbol{C}$, Scatter plots show the effect of APV on the amplitude and duration of the calcium events across the different anatomic regions. Note that blocking NMDA receptors primarily targeted calcium events with faster durations (i.e., $<10$ s). In the medial cortex (MC) from control slices, application of APV eliminated all activity. The $\triangle F / F$ amplitude at the PC in the presence of APV went below the cutoff threshold of $0.01 \Delta \mathrm{F} / \mathrm{F}$; thus, these data points are not shown. Additional details on the statistical analysis are found in Table 1 under the Figure 6 section.

before and after applying PTX, which indicates that the temporal pattern of neural activity was not altered by PTX. Moreover, no ROls in slices from Pyr:Kcnq2 mice exhibited sustained oscillations in the HF band, and this trend remained unaffected by the application of PTX (Fig. 5D)

Combined, Figures 4, 5 indicate that PTX increases the energy of the fluorescence signals from each ROI without 
A

$8 \mathrm{mM} \mathrm{Ko}$
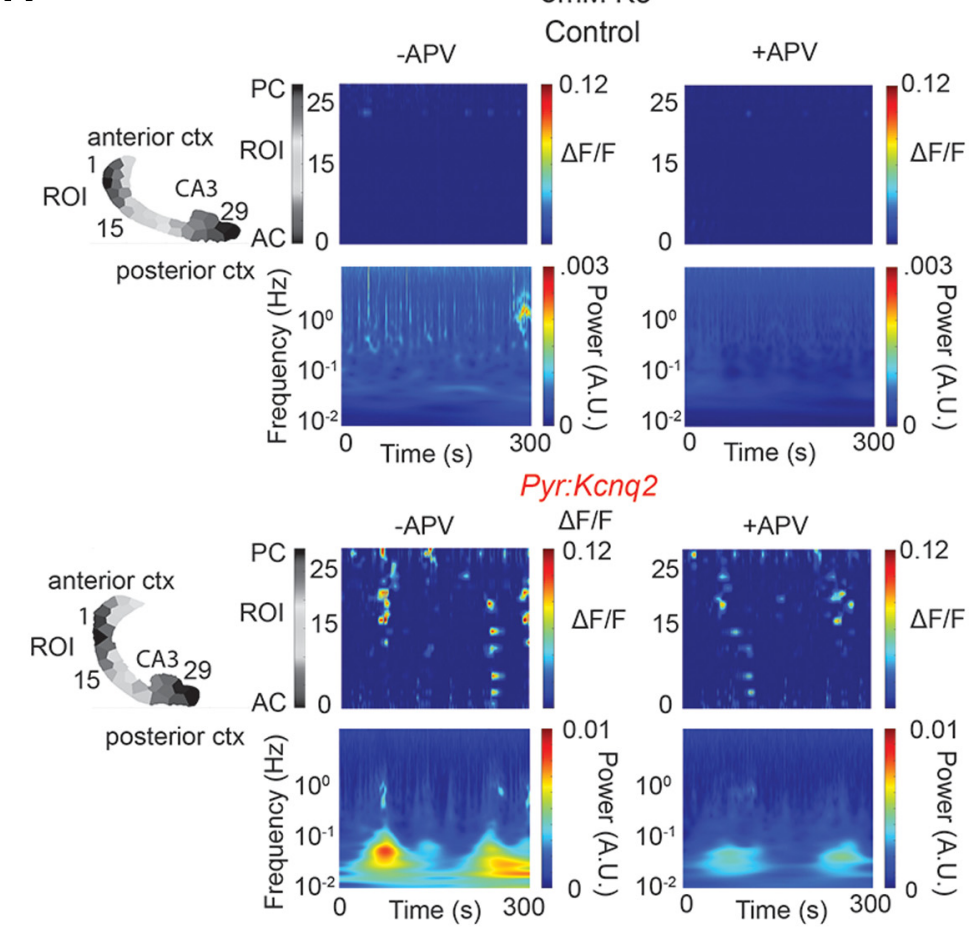

B
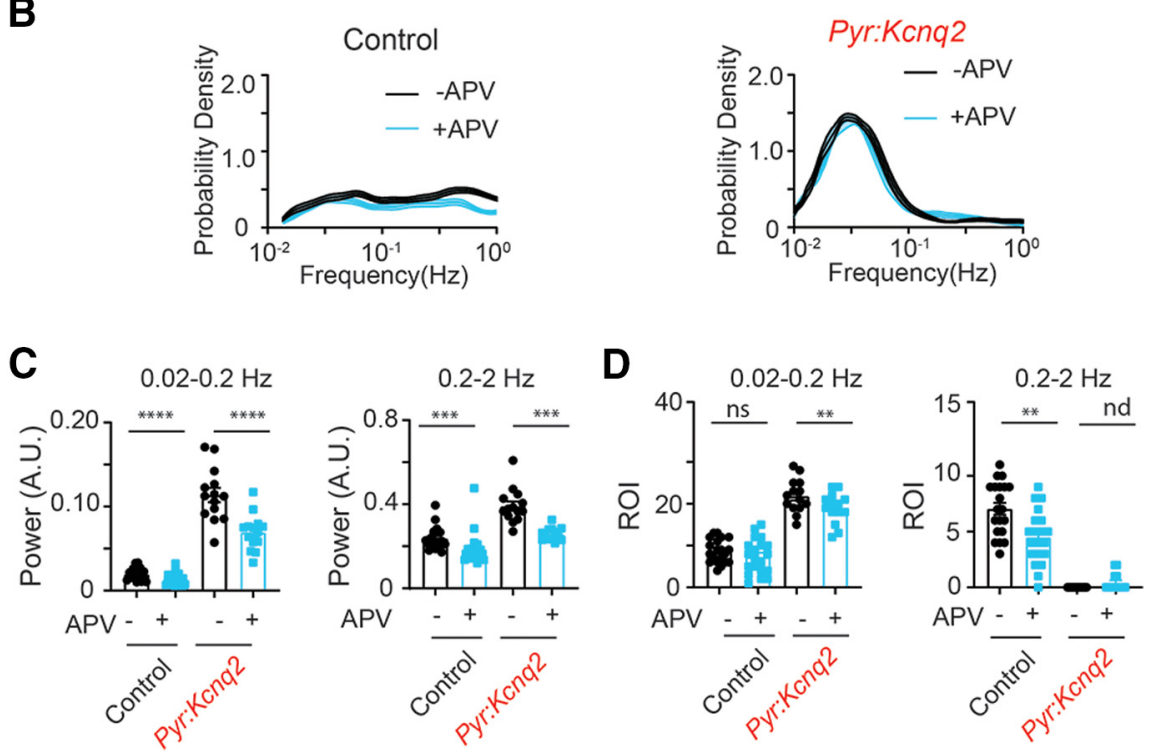

Figure 7. Blocking NMDA receptors does not prevent the emergence of slow oscillatory calcium activity in Pyr:Kcnq2 slices. All recordings were in the presence of $8 \mathrm{~mm}$ Ko. $\boldsymbol{A}$, Representative examples of control and Pyr:Kcnq2 2D plots show calcium activity across ROls and the corresponding wavelets. 2D plots and wavelets before and after application of $25 \mu \mathrm{M}$ D-APV (APV) are shown. Note that APV reduced the power of the slow oscillatory activity in Pyr:Kcnq2 slices. $\boldsymbol{B}$, Comparison of the probability density for the transient oscillation frequency $(f)$ in the presence and absence of APV in control $(n=20)$ and Pyr:Kcnq2 $(n=14)$ hemispheres. Note that APV did not change the likelihood of the emergence of a slow oscillatory activity at the $0.03-$ to $0.05-\mathrm{Hz}$ frequency range. $\boldsymbol{C}$, Summary graphs show the effect of APV in control and Pyr:Kcnq2 hemispheres on the power at the LF (0.02-0.2 Hz) and HF $(0.2-2 \mathrm{~Hz})$ domains. Note that APV reduced the power across all oscillatory frequencies, suggesting that NMDA receptors promote the calcium activity. $\boldsymbol{D}$, Summary graphs show the effect of APV on the number of ROls in control and Pyr:Kcnq2 slices, demonstrating sustained oscillations at frequency $f^{*}$. Note that blocking NMDA receptors led to a decrease in the number of ROls undergoing sustained oscillations in Pyr:Kcnq2 hemispheres, consistent with our observed reduction in power shown in $\boldsymbol{C}$. Data are represented as mean \pm SEM $\left({ }^{* *} p<0.01,{ }^{* \star *} p<0.001,{ }^{* \star *} p<0.0001\right)$. Additional details on the statistical analysis for panel $\boldsymbol{C}$ are found in Table 1 under the Figure 7 section. nd, not determined. 


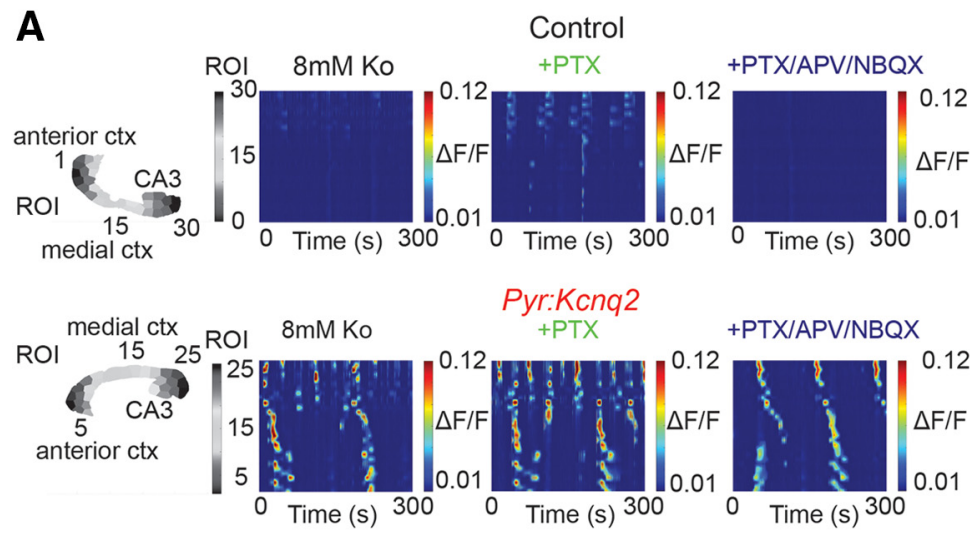

B
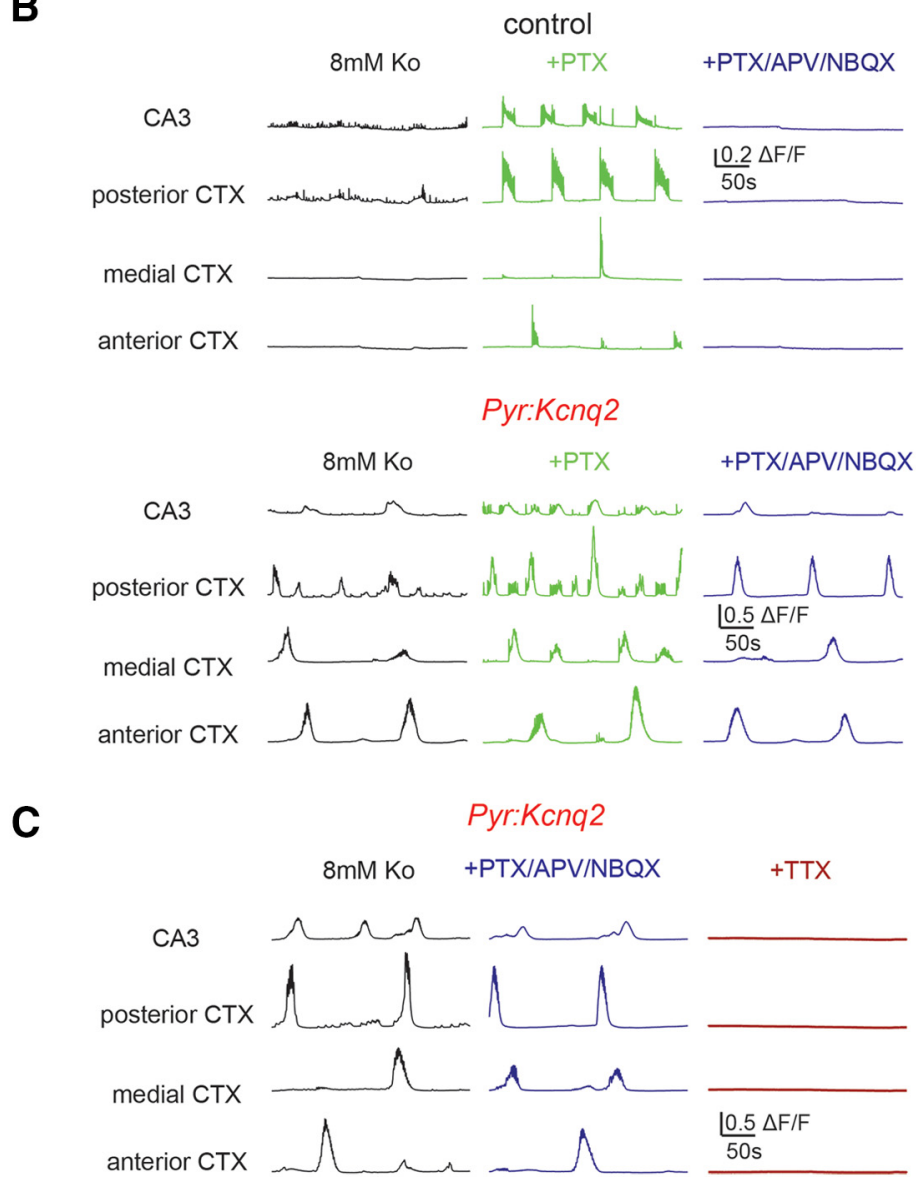

Figure 8. Calcium activity is maintained in the presence of fast excitatory transmission blockers, but eliminated by TTX in slices from Pyr:Kcnq2 mice. All recordings were in the presence of $8 \mathrm{~mm} \mathrm{Ko.} \mathrm{A,} \mathrm{top} \mathrm{panels,} \mathrm{Representative} \mathrm{examples} \mathrm{of} \mathrm{control} \mathrm{and} \mathrm{Pyr:}$ Kcnq2 slices demonstrating calcium activity across all ROls in the presence and absence of $50 \mu \mathrm{M}$ PTX followed by $25 \mu \mathrm{M}$ D-APV and $25 \mu \mathrm{M}$ NBQX. The numbering corresponds to the segmented area shown on the left with lower values toward the anterior cortex $(\mathrm{AC})$ and higher values toward the posterior cortex (PC). B, Calcium activity ( $\triangle \mathrm{F} / \mathrm{F})$ across multiple ROls representing different forebrain anatomic areas in the presence and absence of the GABAA receptor blocker PTX (middle panels) and glutamatergic transmission blockers APV and NBQX (right panels). Note that APV/NBQX primarily inhibited the smaller and faster calcium events that had emerged in the presence of PTX in Pyr:Kcnq2 slices. Summary information regarding the amplitude, duration, and event frequency for multiple slices is found in Table 1 under the Figure 8 section. C, $1 \mu \mathrm{M}$ TTX ( $n=6$ slices) abolishes the calcium responses in slices preincubated with PTX/APV/NBQX.

altering the temporal pattern of the activity, both in slices from Pyr:Kcnq2 and control mice. This finding suggests that the application of PTX facilitates the synchronization among neurons within each ROI (i.e., local microcircuits) but has limited impact on the recruitment of distinct ROIs into a common pattern. It also suggests that the shift in behavior observed in slices from Pyr:Kcnq2 mice is not affected by local synaptic GABAergic transmission but 
rather stems from a change in neural excitability throughout the forebrain.

\section{NMDA receptor inhibition attenuates Kcnq2 slow- wave patterns}

We then assessed whether the LF activity observed in slices from Pyr:Kcnq2 mice depends on glutamatergic activity. Previous work has shown that in the presence of $8 \mathrm{~mm} \mathrm{Ko}$, increased network excitability is partly driven by the activation of NMDA glutamate receptors (Traynelis and Dingledine, 1988). This phenomenon occurs because NMDA receptors have slow deactivation kinetics, which allows for prolonged dendritic depolarization, leads to a downstream increase in somatic and axonal excitability, and causes a buildup of extracellular potassium.

Consistent with this model, application of $25 \mu \mathrm{M}$ D-2amino-5-phosphonovalerate (D-APV) to our control slices reduced the amplitude of the calcium signals across all regions by $50-100 \%$ with the smallest effect at the CA3 region and the largest at the medial cortex (Fig. 6), thus suggesting that NMDA receptor activity is a key driver of network excitability in control slices. In contrast, inhibition of NMDA receptors had more complex effects in slices from Pyr:Kcnq2 mice. First, blocking NMDA receptors reduced calcium activity by $\sim 30-50 \%$ in all forebrain regions (Fig. 6B,C). Second, blocking NMDA receptors prolonged the duration of calcium signals across all regions (Fig. 6C). This was because of the fact that NMDA receptor inhibition primarily targets large, faster events, causing the average signals to be mediated by longer lasting synchronous events (Fig. 6C). Accordingly, the time-frequency signal was significantly attenuated by application of APV (Fig. 7A) and resulted in a significant attenuation (i.e., 20-30\%) of the power content both at LF and HF values (Fig. 7C). However, the application of APV did not alter substantially the sample distribution of transient oscillation frequencies (Fig. 7B) or the number of ROls with significant sustained oscillations (Fig. 7D). Altogether, the behavior described in Figures 6,7 is in contrast to the effect induced by blocking GABAA receptors and suggests that ongoing population network activity in Kcnq2-null excitatory neurons partially requires NMDA receptors. Furthermore, these results suggest that, in Pyr:Kcnq2 slices, NMDA receptor inhibition primarily reduces the overall activity without necessarily suppressing large calcium events.

\section{Fast synaptic transmission does not prevent slow waves in slices from Ppyr:Kcnq2 mice}

To clarify whether the modulatory behavior reported above is restricted to NMDA receptors or rather extends to rapid synaptic transmission in general, we imaged control and Pyr:Kcnq2 slices in the presence of the rapid synaptic transmission blockers 2,3-dioxo-6-nitro-7-sulfamoyl-benzo[f]quinoxaline (NBQX; AMPA receptor blocker), D-APV (NMDA receptor blocker), and PTX (GABAA receptor blocker). We found that blocking all fast synaptic transmission entirely abolished calcium activity in control slices (Fig. $8 A, B$ ), as reported

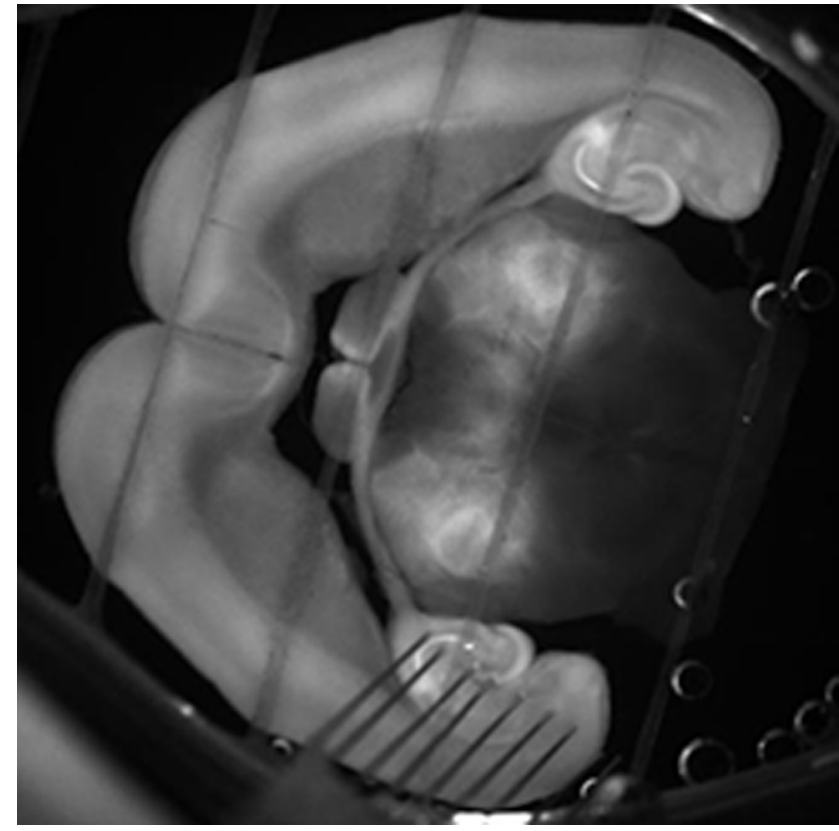

Movie 3. Representative imaging showing the forebrain calcium activity in the absence of Kcnq2 in excitatory neurons and in the presence of synaptic blockers PTX, APV, and NBQX. Movie played at $5 \times$ speed. [View online]

previously (Sipila et al., 2005; Sheroziya et al., 2009). In contrast, in Pyr:Kcnq2 slices activity was reduced across the forebrain, but still persisted significantly (Fig. 8B; Movie 3). In particular, we found a substantial reduction in pulsatile events of smaller amplitude and higher frequency, while large-amplitude calcium population events remained intact. Because of this, we observed an increase in the amplitude (posterior and medial cortex) and duration (CA3 region of the hippocampus, posterior and anterior cortex; Fig. 8B; see Table 1 for data summary). We note that the observed increases in duration and average amplitude were primarily because of the loss of small and short-lasting events; thus, the average duration was enriched by the long-lasting high-amplitude calcium events, rather than the emergence of new larger calcium events. We also did not find any effect of fast synaptic blockers on the propagation velocity of the calcium events $(0.59 \pm 0.11$ $\mathrm{mm} / \mathrm{s}, n=20$ hemispheres; $0.69 \pm 0.13 \mathrm{~mm} / \mathrm{s}, n=18$, $p=0.74$ Mann-Whitney $U$ test). In contrast to the experiments using fast synaptic blockers, calcium responses were eliminated on application of $1 \mu \mathrm{M}$ tetrodotoxin (TTX; Fig. 8C), suggesting that voltagegated sodium channel activity was required for the aberrant calcium responses in Pyr:Kcnq2 slices.

Similar to the data in Figure 8, we observed no significant changes in the time-frequency patterns in the Pyr: Kcnq2 slices on suppression of fast synaptic transmission, i.e., the suppression failed to evoke substantial changes in the frequency distribution of transient oscillations across ROls (Fig. 9A,B) or the number of ROls that exhibited sustained oscillations (Fig. 9D). Instead, blocking fast synaptic transmission lowered the overall intensity 
Table 1: Summary data and statistical analysis for Figures 1-13

\begin{tabular}{|c|c|c|c|c|c|c|c|}
\hline \multirow{2}{*}{$\frac{\text { Figures }}{\text { Figure } 1}$} & & & \multicolumn{3}{|l|}{ Values } & \multicolumn{2}{|l|}{ Statistical tests } \\
\hline & & & & & & & \\
\hline & & & Control & Pyr:Kcnq2 & & & \\
\hline \multirow[t]{14}{*}{$1 B$} & CA3 & Duration & $1.6 \pm 0.46 \mathrm{~s}$ & $2.2 \pm 0.35 \mathrm{~s}$ & Mann-Whitney $U$ test & $p=0.26$ & Control: $n=10 ;$ Pyr: \\
\hline & & & & & & & Kcnq2: $n=18$ \\
\hline & & & & & & & hemispheres \\
\hline & & Events/s & $0.0054 \pm 0.0015$ & $0.013 \pm 0.0025$ & Mann-Whitney $U$ test & $p=0.0143$ & Control: $n=22 ;$ Pyr: \\
\hline & & & & & & & Kcnq2: $n=21$ \\
\hline & & & & & & & hemispheres \\
\hline & Posterior & Duration & $1.15 \pm 0.24 \mathrm{~s}$ & $5.3 \pm 0.61 \mathrm{~s}$ & Mann-Whitney $U$ test & $p<0.0001$ & Control: $n=15 ;$ Pyr: \\
\hline & cortex & & & & & & Kcnq2: $n=20$ \\
\hline & & & & & & & hemispheres \\
\hline & & Events/s & $0.008 \pm 0.0018 \mathrm{~Hz}$ & $0.015 \pm 0.0018 \mathrm{~Hz}$ & Mann-Whitney $U$ test & $p=0.0039$ & Control: $n=22 ;$ Pyr: \\
\hline & & & & & & & Kcnq2: $n=20$ hemi- \\
\hline & & & & & & & spheres \\
\hline & & & & & & & Animals: Control: $n=6$; \\
\hline & & & & & & & Pyr:Kcnq2: $n=6$ \\
\hline \multirow[t]{2}{*}{$1 C$} & CA3 & APs vs current & & & Two-way ANOVA & $(p=0.0025$ & Control: $n=5 ; p P y r:$ \\
\hline & & injections & & & & $\left.F_{(9,33)}=3.745\right)$ & Kcnq2: $n=6$ cells \\
\hline \multicolumn{8}{|l|}{ Figure 2} \\
\hline & & & Control & Pyr:Kcnq2 & Statistical tests and values & & \\
\hline \multirow[t]{17}{*}{$2 C$} & CA3 & Amplitude & $0.064 \pm 0.007 \triangle F / F$ & $0.14 \pm 0.014 \triangle F / F$ & Mann-Whitney $U$ test & $p<0.0001$ & Control: $n=46 ;$ Pyr: \\
\hline & & Duration & $1.4 \pm 0.18 \mathrm{~s}$ & $8.5 \pm 0.68 \mathrm{~s}$ & Mann-Whitney $U$ test & $p<0.0001$ & Kcnq2: $n=56$ \\
\hline & & & & & & & hemispheres \\
\hline & & Events/s & $0.26 \pm 0.023 \mathrm{~Hz}$ & $0.043 \pm 0.0047 \mathrm{~Hz}$ & Mann-Whitney $U$ test & $p<0.0001$ & \\
\hline & Posterior & Amplitude & $0.08 \pm 0.006 \triangle F / F$ & $0.29 \pm 0.023 \triangle F / F$ & Mann-Whitney $U$ test & $p<0.0001$ & Control: $n=44 ;$ Pyr: \\
\hline & cortex & Duration & $2.2 \pm 0.19 \mathrm{~s}$ & $6.5 \pm 0.37 \mathrm{~s}$ & Mann-Whitney $U$ test & $p<0.0001$ & Kcnq2: $n=56$ \\
\hline & $(\mathrm{PC})$ & & & & & & hemispheres \\
\hline & & Events/s & $0.14 \pm 0.0072 \mathrm{~Hz}$ & $0.048 \pm 0.0032 \mathrm{~Hz}$ & Mann-Whitney $U$ test & $p<0.0001$ & \\
\hline & Medial & Amplitude & $0.021 \pm 0.01 \triangle \mathrm{F} / \mathrm{F}$ & $0.33 \pm 0.029 \triangle F / F$ & Mann-Whitney $U$ test & $p<0.0001$ & Control, $n=43 ;$ Pyr: \\
\hline & cortex & Duration & $1.4 \pm 0.58 \mathrm{~s}$ & $9.5 \pm 0.56 \mathrm{~s}$ & Mann-Whitney $U$ test & $p<0.0001$ & Kcnq2: $n=55$ \\
\hline & (MC) & & & & & & hemispheres \\
\hline & & Events/s & $0.0065 \pm 0.0024 \mathrm{~Hz}$ & $0.018 \pm 0.0023 \mathrm{~Hz}$ & Mann-Whitney $U$ test & & \\
\hline & Anterior & Amplitude & $0.02 \pm 0.003 \triangle \mathrm{F} / \mathrm{F}$ & $0.36 \pm 0.03 \triangle F / F$ & Mann-Whitney $U$ test & $p<0.0001$ & Control: $n=44 ;$ Pyr: \\
\hline & cortex & Duration & $1.5 \pm 0.3 \mathrm{~s}$ & $11.2 \pm 0.8 \mathrm{~s}$ & Mann-Whitney $U$ test & $p<0.0001$ & Kcnq2: $n=54$ \\
\hline & $(\mathrm{AC})$ & & & & & & hemispheres \\
\hline & & Events/s & $0.012 \pm 0.0021 \mathrm{~Hz}$ & $0.021 \pm 0.0025 \mathrm{~Hz}$ & Mann-Whitney $U$ test & $p=0.0005$ & \\
\hline & & & Data & re obtained from 8 con & ol and 9 Pyr:Kcnq2 mice. & & \\
\hline \multicolumn{8}{|l|}{ Figure 3} \\
\hline & & & Control & Pyr:Kcnq2 & Statistical tests and values & & \\
\hline \multirow[t]{3}{*}{$3 C$} & Power 0.0 & $0.2 \mathrm{~Hz}$ & $0.022 \pm 0.0015$ A.U. & $0.11 \pm 0.0046$ A.U. & Mann-Whitney $U$ test & $p<0.0001$ & Control, $n=43 ;$ Pyr: \\
\hline & Power 0.2 & & $0.026 \pm 0.011$ A.U. & $0.38 \pm 0.012 A . U$ & Mann-Whitney $U$ test & $p<0.0001$ & Kcnq2:, $n=47$ \\
\hline & & & & & & & hemispheres \\
\hline \multirow[t]{3}{*}{$3 D$} & $\mathrm{ROI} 0.02-\mathrm{C}$ & $\mathrm{Hz}$ & $10.1 \pm 0.7 \mathrm{ROls}$ & $19.9 \pm 0.7 \mathrm{ROls}$ & Mann-Whitney $U$ test & $p<0.0001$ & Control, $n=43 ;$ Pyr: \\
\hline & $\mathrm{ROI} 0.2-2$ & & $6.6 \pm 0.5 \mathrm{ROls}$ & $0.5 \pm 0.2 \mathrm{ROls}$ & Mann-Whitney $U$ test & $p<0.0001$ & Kcnq2:, $n=47$ \\
\hline & & & & & & & hemispheres \\
\hline \multicolumn{8}{|c|}{ Data were obtained from 8 control and 9 Pyr:Kcnq2 mice. } \\
\hline \multicolumn{8}{|l|}{ Figure 4} \\
\hline & & & & Control & Pyr:Kcnq2 & Statistical tests and values & \\
\hline \multirow[t]{14}{*}{$4 C$} & CA3 & Amplitude & -PTX $0.057 \triangle \mathrm{F} /$ & $-\mathrm{PTX} 0.08 \triangle \mathrm{F} /$ & Mann-Whitney $U$ test & Control & Control: -PTX: $n=1547$ \\
\hline & & & $F \pm 0.055 S D$ & $F \pm 0.09 S D$ & & $p<0.0001$ & +PTX: $n=1322$ events \\
\hline & & & $+\mathrm{PTX} 0.27 \triangle \mathrm{F} /$ & $+\mathrm{PTX} 0.19 \triangle \mathrm{F} /$ & Mann-Whitney $U$ test & Pyr:Kcnq2 & Pyr:Kcnq2: -PTX: $n=224$ \\
\hline & & & $F \pm 0.12 S D$ & $F \pm 0.09 S D$ & & $p<0.0001$ & events ; +PTX: $n=523$ \\
\hline & & & & & & & events \\
\hline & & Duration & $-\mathrm{PTX} 0.8 \mathrm{~s} \pm 1.5 \mathrm{SD}$ & $-\mathrm{PTX} 4.3 \mathrm{~s} \pm 5.4 \mathrm{SD}$ & Mann-Whitney $U$ test & Control & \\
\hline & & & & & & $p<0.0001$ & \\
\hline & & & $+\mathrm{PTX} 0.7 \pm 1.7 \mathrm{SD}$ & $+\mathrm{PTX} 1.8 \pm 3.8 \mathrm{SD}$ & Mann-Whitney $U$ test & Pyr:Kcnq2 & \\
\hline & & & & & & $p<0.0001$ & \\
\hline & Posterior & Amplitude & -PTX $0.08 \triangle \mathrm{F} /$ & $-\mathrm{PTX} 0.21 \triangle \mathrm{F} /$ & Mann-Whitney $U$ test & Control & Control: -PTX: $n=931$; \\
\hline & cortex & & $F \pm 0.058 S D$ & $F \pm 0.26 S D$ & & $p<0.0001$ & +PTX: $n=777$ events \\
\hline & & & $+\mathrm{PTX} 0.43 \triangle \mathrm{F} /$ & $+\mathrm{PTX} 0.38 \triangle \mathrm{F} /$ & Mann-Whitney $U$ test & Pyr:Kcnq2 & Pyr:Kcnq2: -PTX: $n=223$; \\
\hline & & & $F \pm 0.20 S D$ & $F \pm 0.24 S D$ & & $p<0.0001$ & +PTX: $n=444$ events \\
\hline & & & & (Cor & & & \\
\hline
\end{tabular}


Table 1: Continued

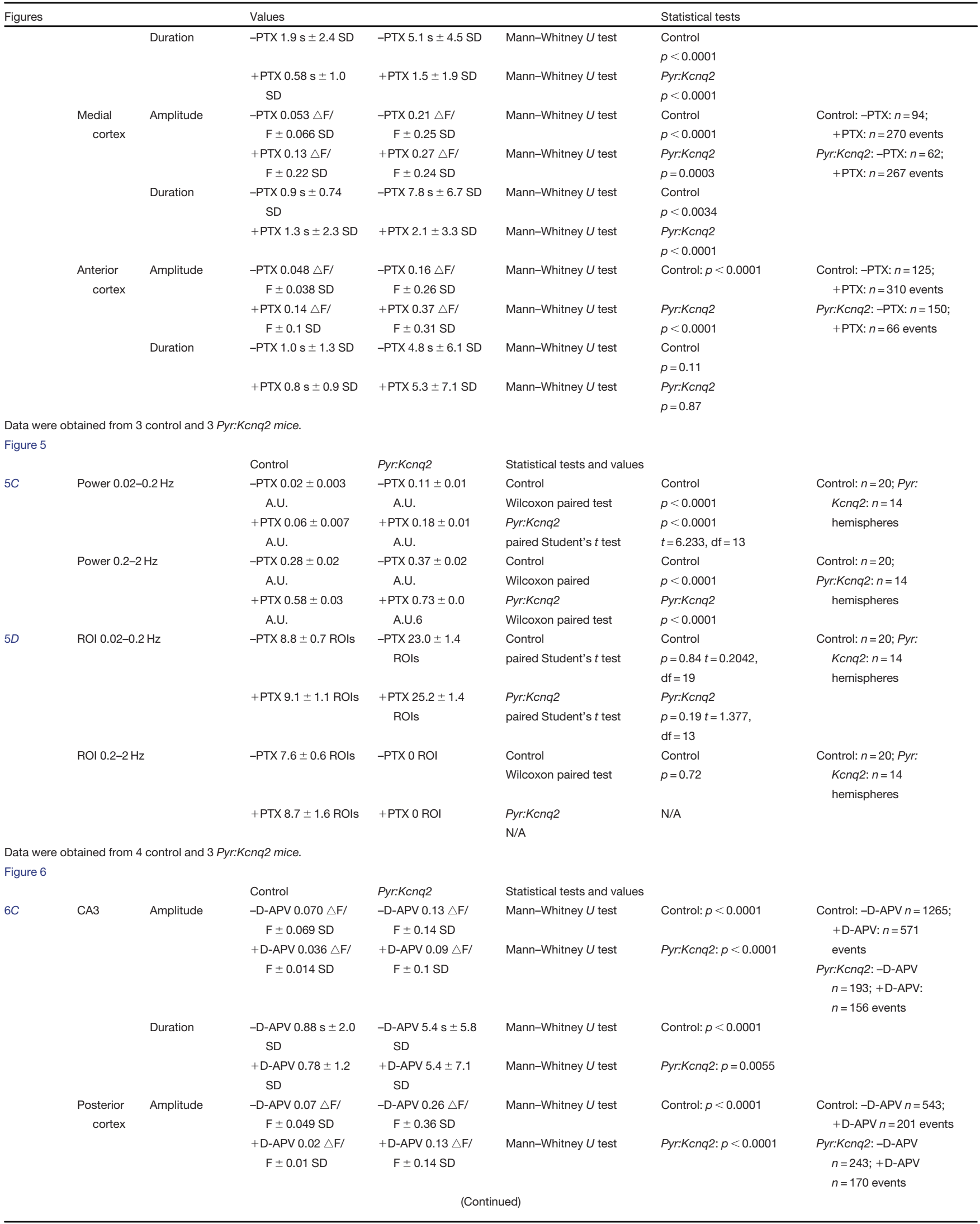


Table 1: Continued

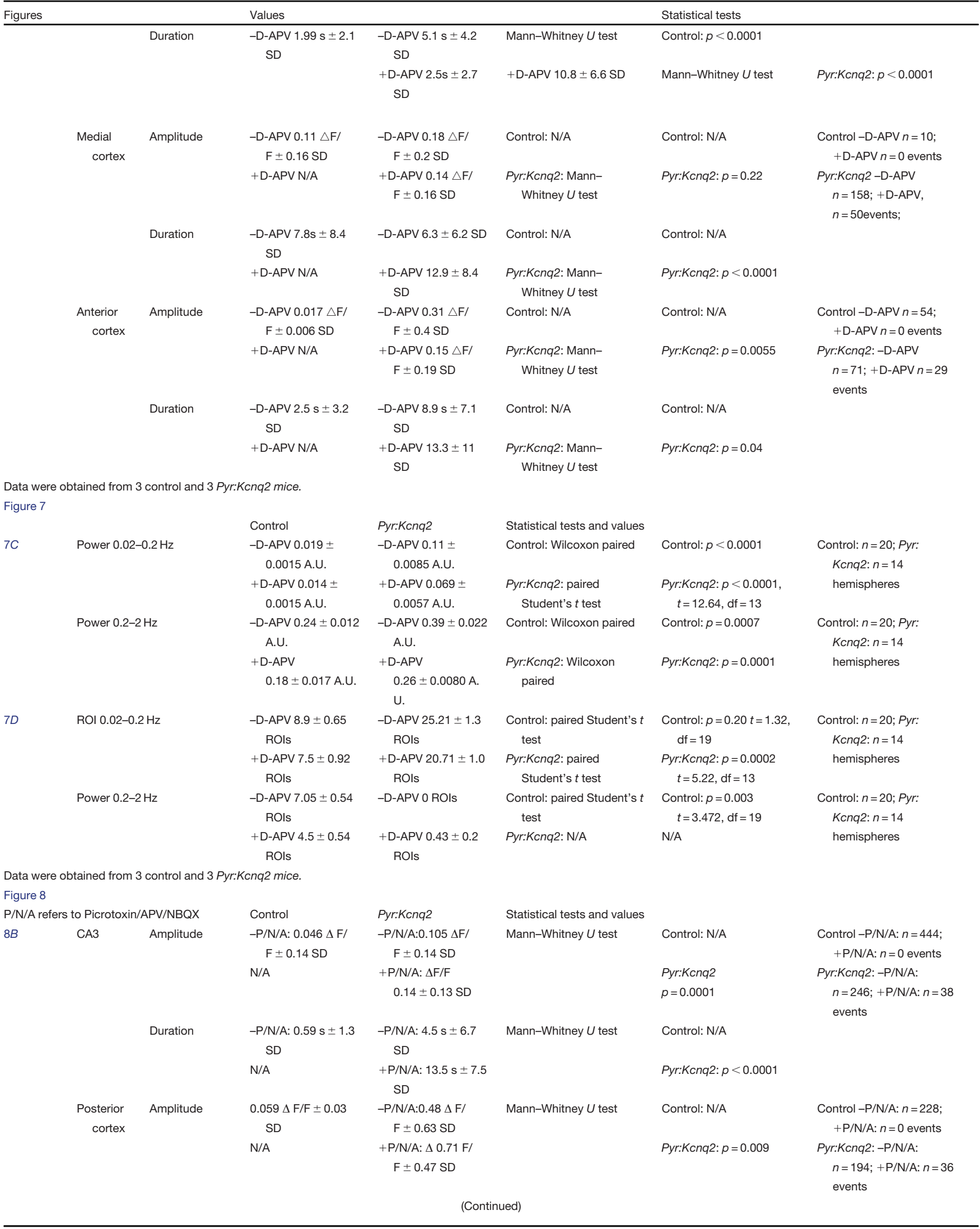


Table 1: Continued

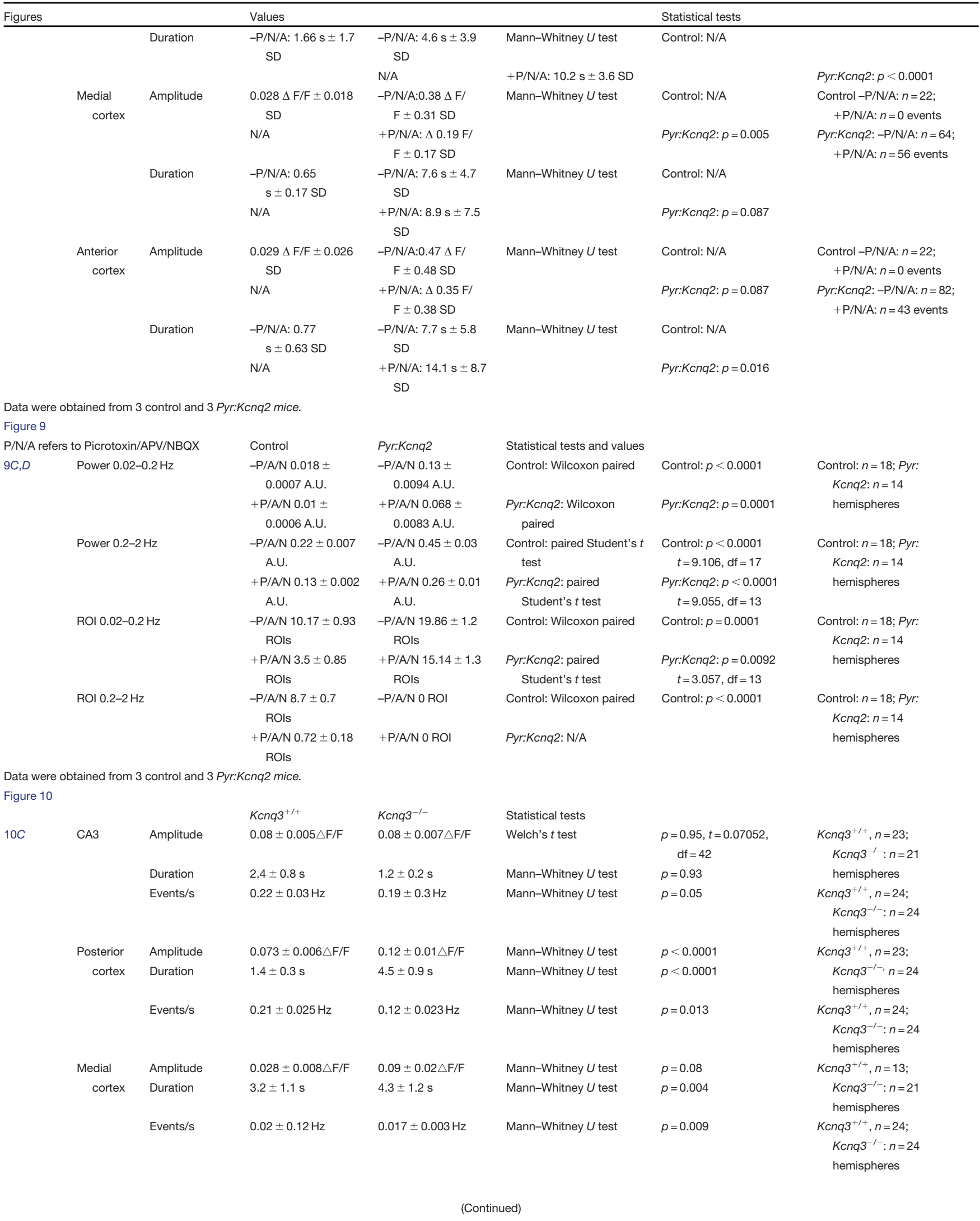


Table 1: Continued

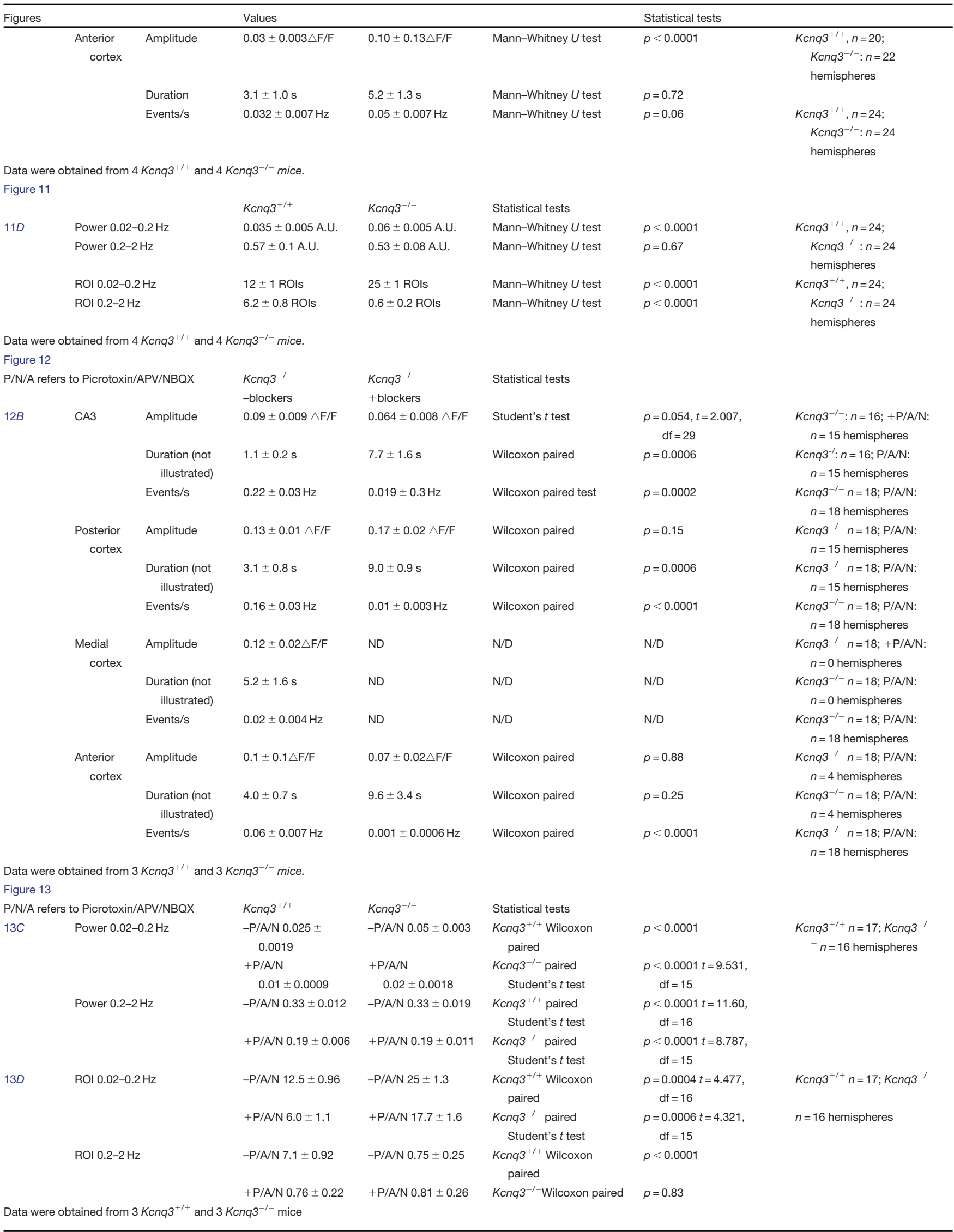




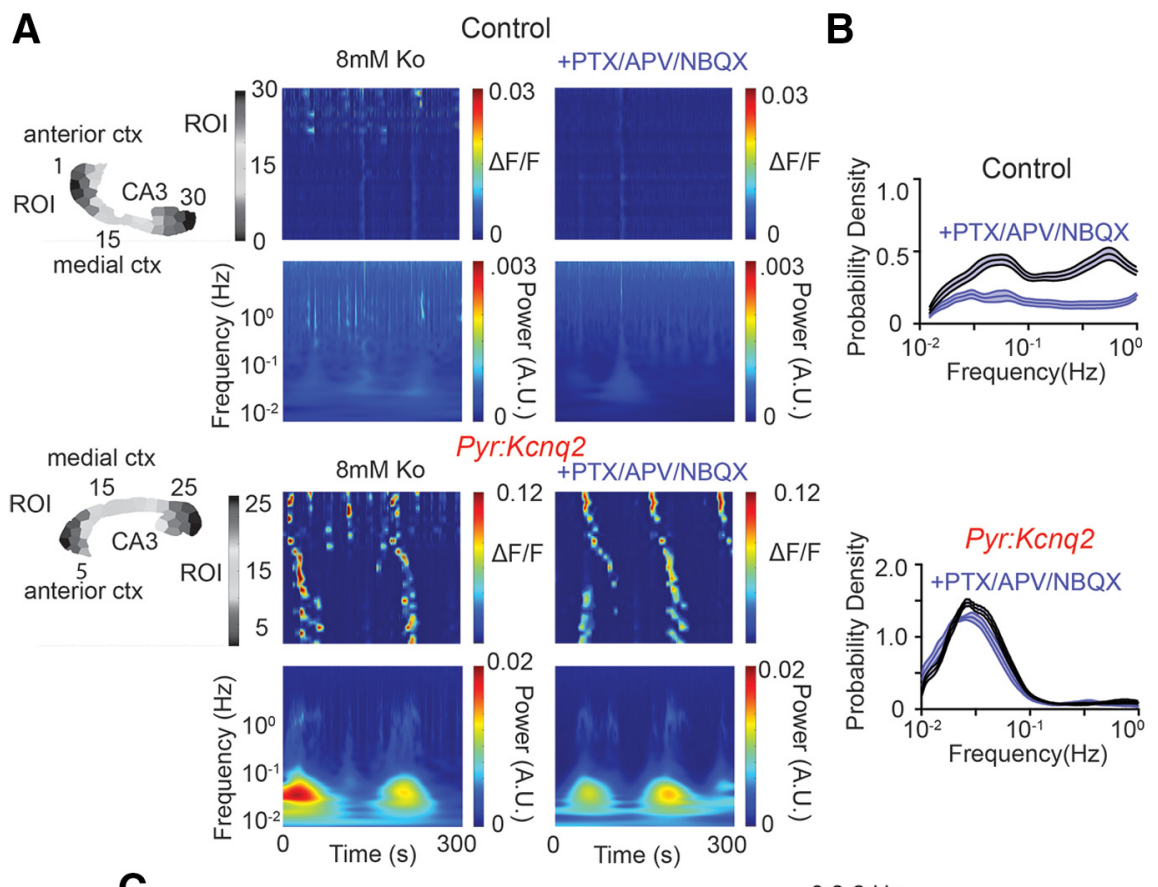

C
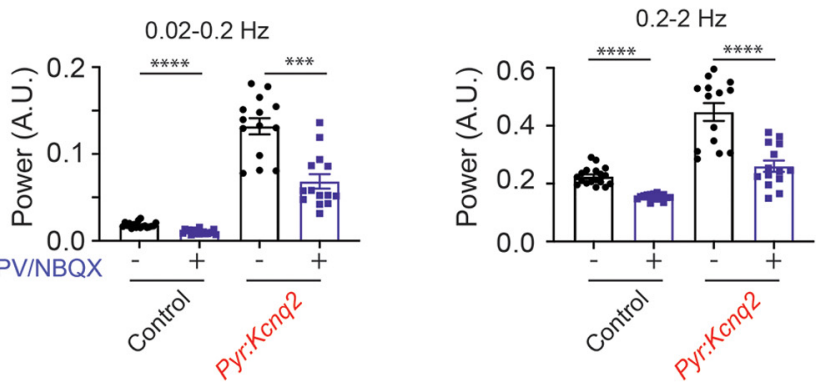

D
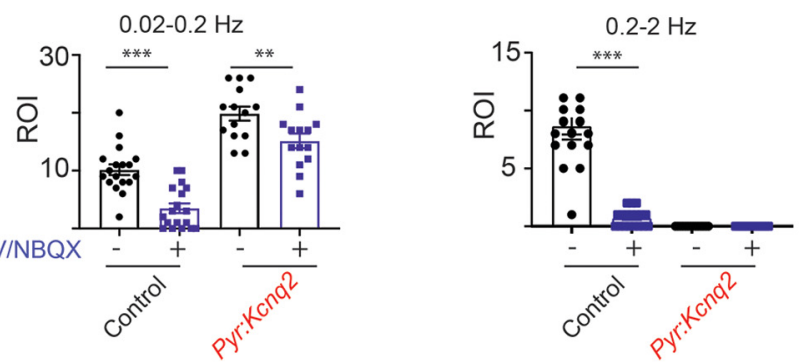

Figure 9. Differential effects of fast synaptic receptor blockers on the oscillation frequencies in control and Pyr:Kcnq2 slices. All recordings were in the presence of $8 \mathrm{~mm}$ Ko. $\boldsymbol{A}$, Representative examples of control and Pyr:Kcnq2 2D plots and their corresponding wavelets in the presence and absence of $50 \mu \mathrm{m}$ PTX, $25 \mu \mathrm{m}$ D-APV, and $25 \mu \mathrm{m}$ NBQX (PTX/APV/NBQX). Note that application of all synaptic blockers reduced the power at low frequencies, but did not prevent the emergence of slow oscillatory activity in the Pyr:Kcnq2 hemisphere. B, Comparison of the probability density of the frequency $f$ of transient oscillations before and after application of PTX/APV/NBQX in control $(n=18)$ and Pyr:Kcnq2 $(n=14)$ hemispheres. $\boldsymbol{C}$, Summary graphs show the effect of PTX/APV/NBQX on the power in control and Pyr:Kcnq2 hemispheres for the LF $(0.02-0.2 \mathrm{~Hz})$ and HF (0.2$2 \mathrm{~Hz}$ ) domains. $\boldsymbol{D}$, Summary graphs show the effect of PTX/APV/NBQX on the number of ROls undergoing sustained oscillations in control and Pyr:Kcnq2 hemispheres. Data are presented as mean \pm SEM $\left({ }^{* *} p<0.01,{ }^{* * *} p<0.001,{ }^{* * * *} p<0.0001\right)$. Additional details on the statistical analysis for panels $\boldsymbol{C}, \boldsymbol{D}$ are found in Table 1 under the Figure 9 section.

of the transient oscillations (Fig. 9A) and resulted in lower spectral content in both the LF and HF bands (Fig. 9C). Finally, we did not observe these effects in the control slices; instead and in line with the expectations of blocking synaptic transmission, we found a robust suppression of transient oscillations across all ROls (Fig. 9B) and a large reduction in the number of ROls exhibiting sustained oscillations (Fig. 9D). Together, these data suggest that loss of KCNQ2 channels drives LF activity through a mechanism that is independent of fast synaptic transmission. 
A

$\mathrm{KCnq3}^{+/+}$

$8 \mathrm{mM} \mathrm{Ko}$

$\mathrm{KCnq}^{-/-}$

anterior CTX
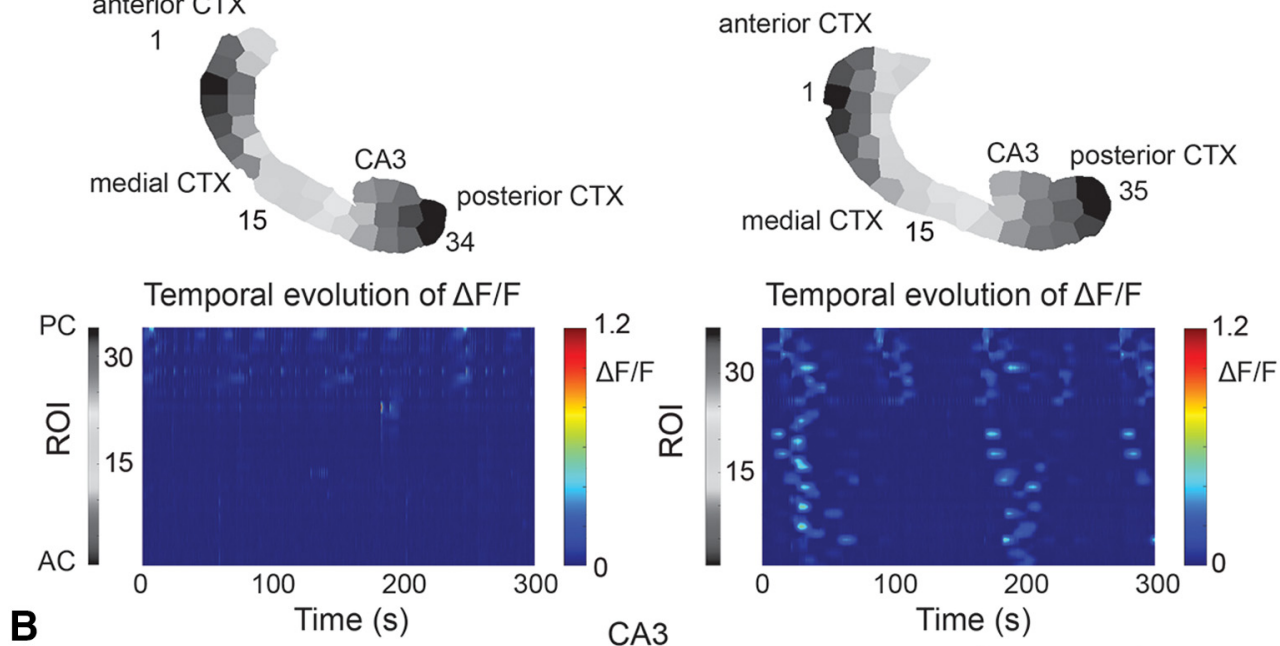

B

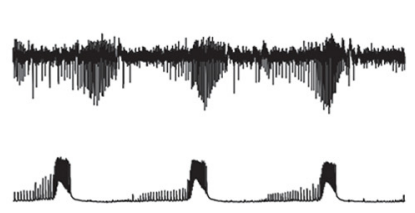

$\frac{\lfloor 100 \mu \mathrm{V}}{50 \mathrm{~s}} \frac{\lfloor 200 \mu \mathrm{V}}{50 \mathrm{~s}}$

CA3

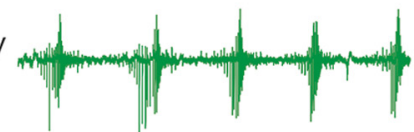

posterior cortex
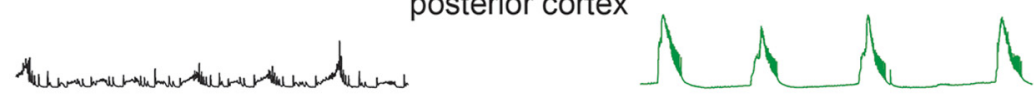

medial cortex

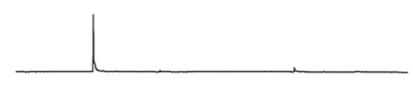

anterior cortex
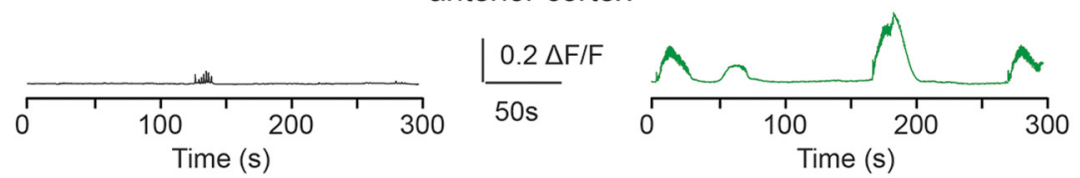

C

$\mathrm{KCnq3}^{+/+}$
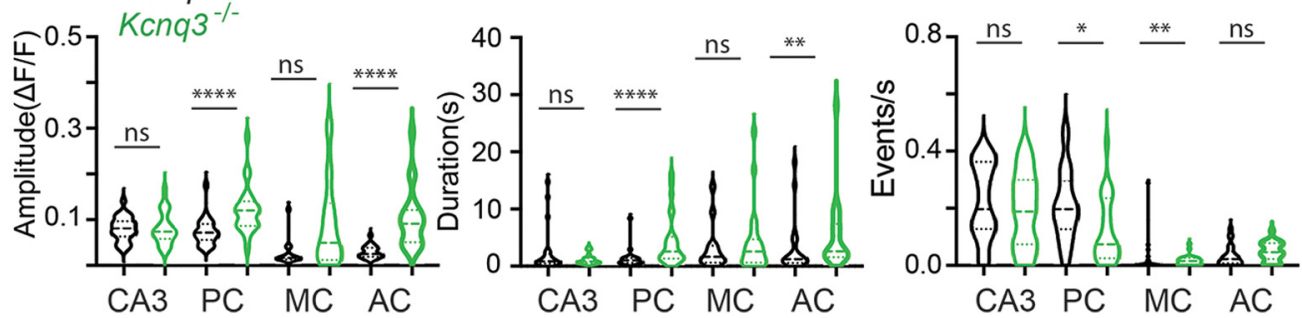

Figure 10. Ablation of $K c n q 3$ leads to hyperexcitability across the forebrain in the presence of 8 mM Ko. A, top panels, Two examples of acute slices from $\mathrm{Kcnq}^{+/+}$and $\mathrm{Kcnq}^{-{ }^{-l-}}$ mice with one hemisphere segmented into ROls. Below, $2 \mathrm{D}$ plots show the changes in the $\Delta \mathrm{F} /$ $\mathrm{F}$ as a function of time for the different ROls. Note that calcium activity is recorded across all regions in the Kcnq3-null slice. $\boldsymbol{B}$, Raw traces show calcium activity $(\Delta F / F)$ across the different anatomic areas. Top two panels, Temporal evolution of LFPs and $\Delta F / F$ recorded in parallel from the CA3 region of the hippocampus. $\boldsymbol{C}$, Violin plots show the effect of Kcnq3 deletion on the $\Delta \mathrm{F} / \mathrm{F}$ amplitude, duration, and event frequency (events/s) for the different anatomic regions. The median and the interquartile ranges are also shown $\left({ }^{*} p<0.05,{ }^{* *} p<0.01\right.$, $\left.{ }^{* \star \star} p<0.0001\right)$. Additional details on the statistical analysis for panel $\mathbf{C}$ are found in Table 1 under the Figure 10 section.

Thus, in the absence of rapid synaptic transmission, slices from Pyr:Kcnq2 mice can still generate large hypersynchronous events, likely because of sodium channeldriven depolarization of Kcnq2-null excitatory neurons and a large build-up and diffusion of potassium (Aiba and Noebels, 2021).

\section{Effects of $K c n q 3$ deletion on network activity}

Recent work has shown that KCNQ3 dysfunction leads to pharmaco-dependent epilepsy in patients lacking both functional Kcnq3 copies (Lauritano et al., 2019), which is distinct from the pharmaco-resistant epilepsy caused by KCNQ2 dominant-negative variants (Cornet et al., 2018). 
Hence, we investigated whether loss of KCNQ3 activity leads to similar or different slow hypersynchronous events in the forebrain compared with loss of KCNQ2 activity. We thus repeated our experiments in Kcnq3-knock-out mice crossed with Emx1 ${ }^{\mathrm{cre}}: \mathrm{PC}: \mathrm{Gcamp5}$, as $\mathrm{Kcnq}^{-1-}$ mice survive to adulthood.

The activity in the Kcnq3-null slices was qualitatively similar to that in the slices without Kcnq2. Thus, ablation of Kcnq3 also increases the network excitability across the forebrain and can lead to the emergence of large LF propagation calcium events $\left(\mathrm{Kcnq}^{-/-}\right.$calcium propagation velocity $=0.73 \pm 0.15 \mathrm{~mm} / \mathrm{s}, n=20$ hemispheres; Fig. 10; Movie 4), thus suggesting a previously unappreciated role of KCNQ3 channels in the neonatal forebrain. However, we note several similarities and differences between the two mouse lines. (1) Unlike in Pyr:Kcnq2 mice, ablation of Kcnq3 did not increase the amplitude or the duration of calcium signals across all areas (Fig. 10B,C). For instance, we primarily found increases in the posterior cortex with less of an effect in the CA3 region of the hippocampus. (2) We observed an increase in the probability density, power, and ROls primarily at the LF domain in $\mathrm{Kcnq3}^{-1-}$ slices. However, the overall increases were smaller than we observed in the Pyr:Kcnq2 slices (compare Figs. 11 and 3). This smaller change in excitability because of Kcnq3 ablation is consistent with the less severe phenotypes of Kcnq3-deficient than Kcnq2-deficient mice (Soh et al., 2014).

Next, we tested whether the increase in LF calcium events in Kcnq3-knock-out mice is dependent on fast synaptic transmission. We found that the neocortical activity in Kcnq3-null mice was fully dependent on fast glutamatergic transmission, as co-application of NBQX and APV in the presence of PTX eliminated all ongoing activity at the neocortex (Fig. 12), this effect was not observed with Pyr:Kcnq2 mice but was similar to what we found in control slices. Consequently, we found an overall decrease in the power and ROIs in slices from either $\mathrm{Kcnq}^{+/+}$or Kcnq3 $3^{-/-}$slices (Fig. 13). Importantly, glutamatergic activity did not block the LF calcium events in the hippocampus similar to what we found in slices from Pyr:Kcnq2 mice. This is best illustrated in Figure 13. Thus, KCNQ2 and KCNQ3 loss could lead to the generation of LF hypersynchronous events, but their dependence on fast synaptic transmission is specific to the forebrain region (see Discussion).

\section{Discussion}

In this work, we determined the role of KCNQ2 and KCNQ3 channels in regulating the network activity of forebrain neonatal neuronal circuits. Although KCNQ2 and KCNQ3 channels are expressed in both GABAergic and glutamatergic neurons (Cooper et al., 2001), we primarily focused on forebrain excitatory cells as KCNQ2/3 channels regulate multiple facets of their excitability. The key findings of our study are that (1) KCNQ2 channel loss from pyramidal neurons can lead to multifocal spreading activity across all regions of the forebrain, (2) large LF calcium events modestly depend on fast synaptic transmission, and (3) unlike Pyr:Kcnq2 mice, Kcnq3-null mice

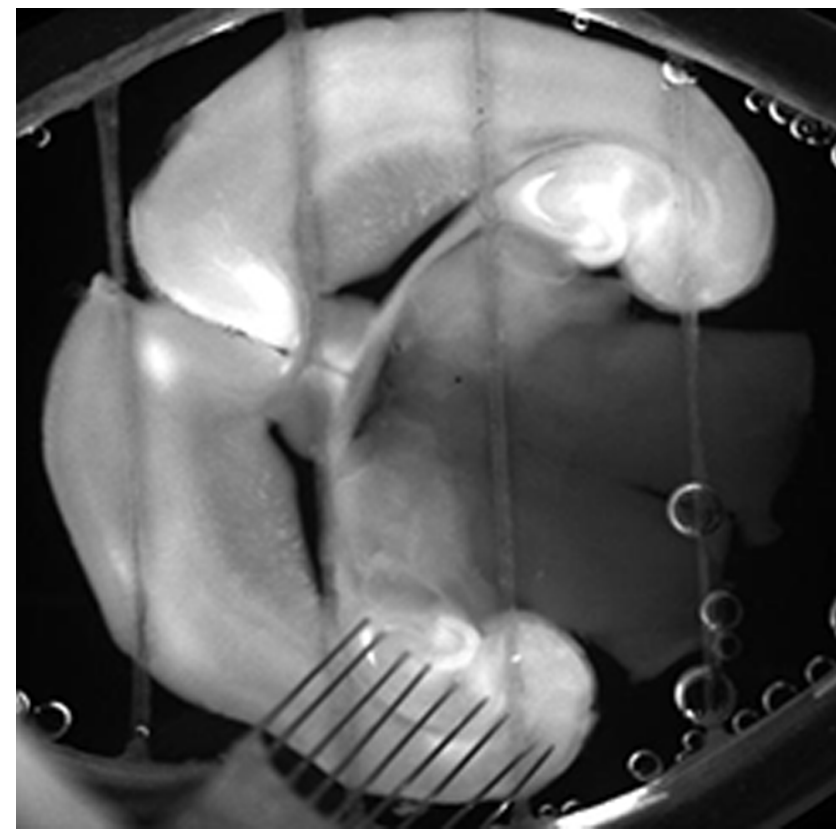

Movie 4. Representative imaging showing the forebrain calcium activity in the absence of Kcnq3. Movie played at $5 \times$ speed. [View online]

exhibit LF hypersynchronous activity driven by glutamatergic transmission in the neocortex but not the hippocampus. Our results provide new insights into the role of KCNQ2 and KCNQ3 channels in the neonatal brain and demonstrate that loss of KCNQ2 channels drives hyperexcitability in a manner distinct from KCNQ3-induced hypersynchrony in the neonatal neocortex.

\section{KCNQ2 channels in the developing forebrain}

KCNQ2 channels are unique as they are expressed early in development, before birth (Kanaumi et al., 2008). For instance, studies examining KCNQ2 mRNA from human brain at different gestational periods have shown that KCNQ2 mRNA is expressed as early as $50 \mathrm{~d}$ postconception (http:// development.psychencode.org/\#). Consistent with this, KCNQ2 protein levels are highly expressed during the perinatal and early infantile period (Kanaumi et al., 2008). Thus, in retrospect it is not surprising that KCNQ2 pathogenic variants lead to range of pediatric epilepsy disorders from benign familial neonatal seizures to DEEs (Nappi et al., 2020).

The critical function of KCNQ2 channels early in development has also been supported in work with certain mouse models. For instance, loss-of-function Kcnq2 homozygous mice die within $1 \mathrm{~h}$ following birth (Watanabe et al., 2000). Additionally, overexpression of dominantnegative isoforms of KCNQ2 channels during the first week of life, but not later, leads to lifelong seizures and premature death (Peters et al., 2005). Our work builds on these previous findings and makes several new contributions. Until now, most studies on the function of KCNQ2 channels in the developing cortex have focused on the hippocampus using pan-KCNQ channel blockers like XE991 and linopiridine (Okada et al., 2003; Qiu et al., 
A

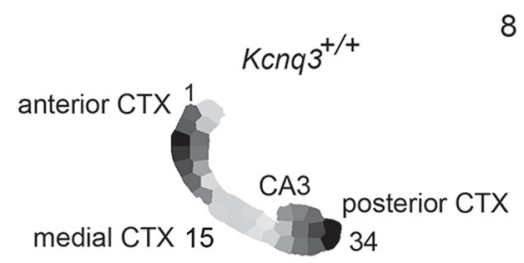

$8 \mathrm{mM} \mathrm{Ko}$

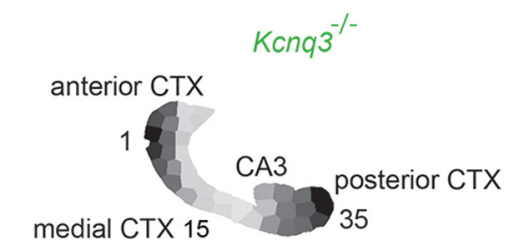

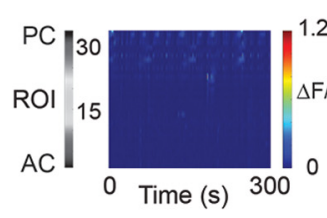
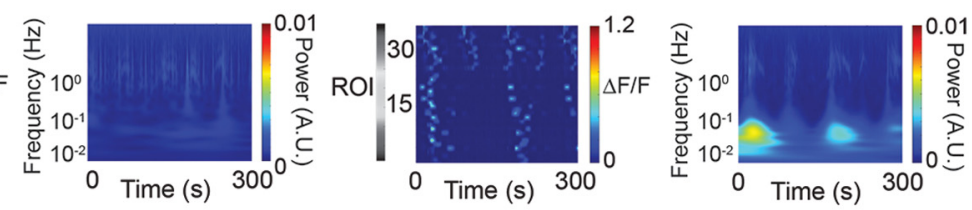

B
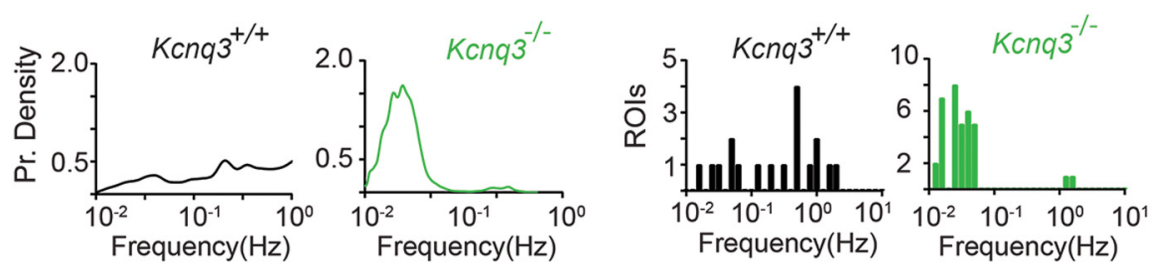

C
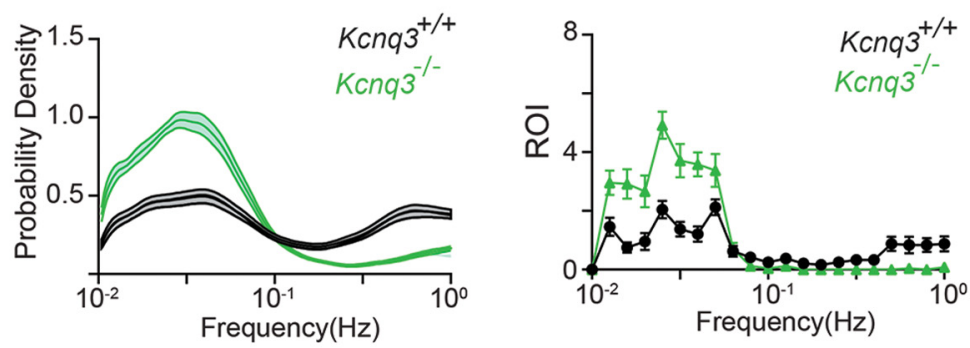

D
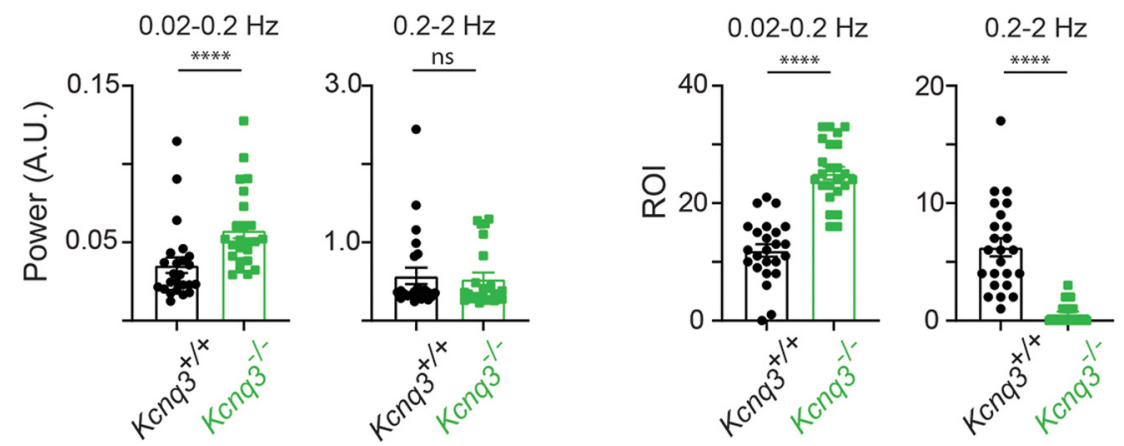

Figure 11. Loss of Kcnq3 leads to LF calcium oscillations in multiple ROls. All recordings were in the presence of 8 mm Ko. $\boldsymbol{A}$, Representative examples of $K c n q 3^{+/+}$and $K c n q 3^{-/-} \Delta \mathrm{F} / \mathrm{F} 2 \mathrm{D}$ plots and wavelets. Top panels, ROls used to generate the $\Delta \mathrm{F} / \mathrm{F} 2 \mathrm{D}$ plots and the wavelets. $\boldsymbol{B}$, Comparison of the probability density of the frequency $f$ of transient oscillations and number of ROls undergoing sustained oscillations for the examples shown in panel $\boldsymbol{A}$. $\boldsymbol{C}$, left, Comparison of the probability density of the frequency $f$ of transient oscillations for $\mathrm{Kcnq}^{+/+}(n=24)$ and $\mathrm{Kcnq}^{-/-}(n=24)$ across multiple hemispheres. Right, Comparison of ROls undergoing sustained oscillations for $\mathrm{Kcnq}^{+/+}(n=24)$ and $\mathrm{Kcnq}^{-/-}(n=24)$ hemispheres at different frequencies. $\boldsymbol{D}$, left panels, Summary graphs of the power at two frequency domains, $0.02-0.2$ and $0.2-2 \mathrm{~Hz}$. Right panels, Summary graphs of the ROls exhibiting sustained oscillations at frequency $f^{*}$ in the range of $0.02-0.2$ and $0.2-2 \mathrm{~Hz}$. Data are represented as mean \pm SEM $\left(^{* * \star} p<\right.$ 0.0001). Additional details on the statistical analysis for panel $\boldsymbol{D}$ are found in Table 1 under the Figure 11 section.

2007; Safiulina et al., 2008). However, such pharmacological reagents could not distinguish between KCNQ2 and KCNQ3, or parse through the contribution of different cell populations. By combining the use of Kcnq2 conditional knock-out mice and mesoscale calcium imaging, we were able to define the function of KCNQ2 channels from excitatory neurons in the neonatal forebrain. Although activity under basal conditions was primarily confined to the 


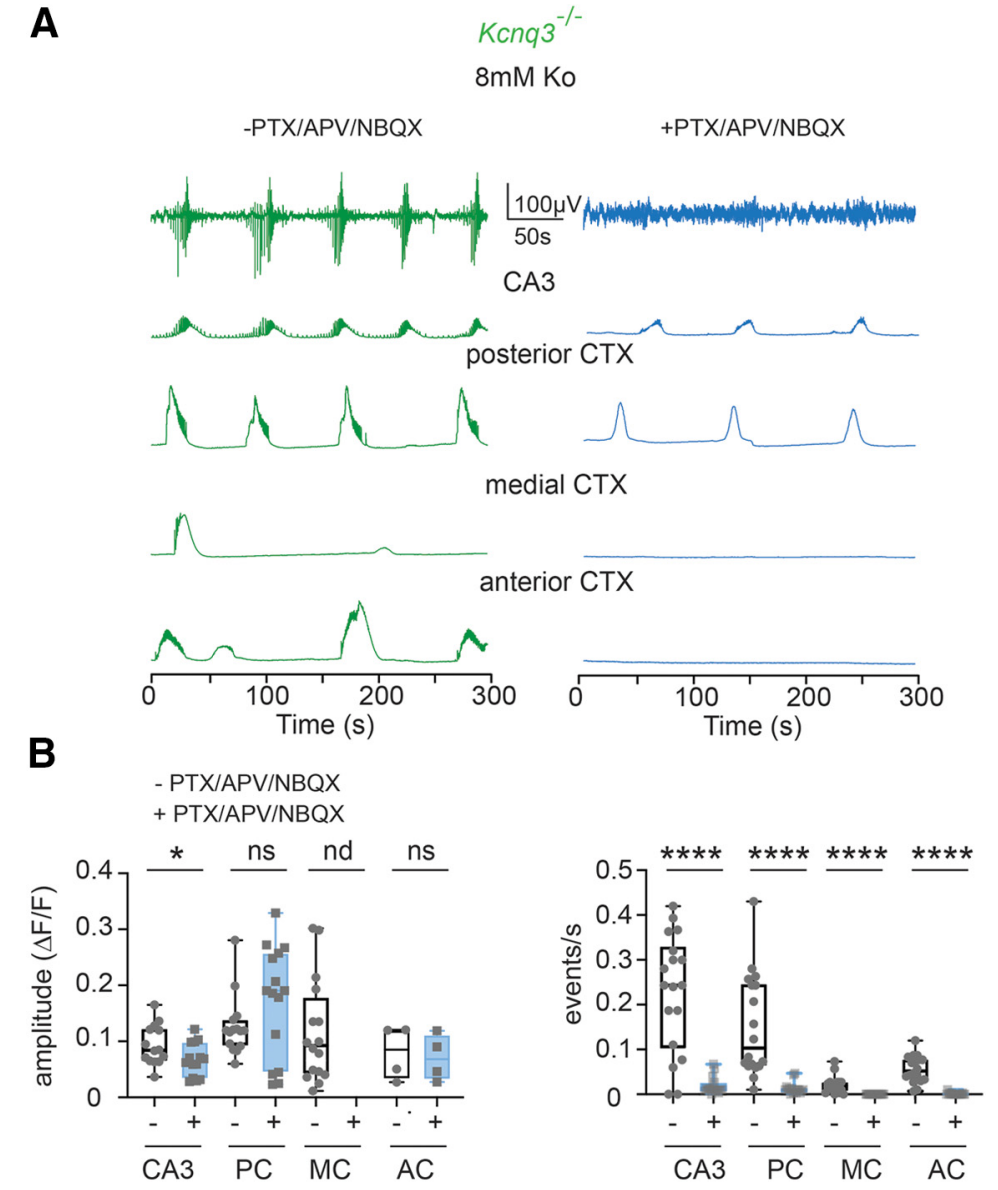

B

Figure 12. Synaptic blockers inhibit calcium activity in a region-specific manner in $\mathrm{Kcnq}^{-1-}$ slices. All recordings were in the presence of $8 \mathrm{~mm}$ Ko. $\boldsymbol{A}$, top two panels, Recorded LFP and $\triangle F / F$ activity from the CA3 region of the hippocampus. Middle and bottom panels, Temporal evolution of the $\Delta F / F$ across different anatomic areas. Note that synaptic blockers (PTX/APV/NBQX) abolish the calcium activity only in the medial cortex (MC) and anterior cortex (AC). B, Box plots show the effect of $K c n q 3^{-1-}$ ablation on the $\Delta \mathrm{F} / \mathrm{F}$ amplitude, and event frequency for the different anatomic regions $\left({ }^{*} p<0.05,{ }^{\star \star \star *} p<0.0001\right)$. Note that although application of synaptic blockers did not inhibit the amplitude of calcium events equally across the different regions, it did lead to a decrease to the number of calcium events across all regions. Additional details on the statistical analysis and number of replicates for panel $\boldsymbol{B}$ are found in Table 1 under the Figure 12 section.

hippocampus and posterior cortex, application of $8 \mathrm{~mm}$ extracellular potassium revealed a large hyperexcitable phenotype across the forebrain in slices from Pyr:Kcnq2 animals. The activity was multifocal, that is multiple regions showed large population calcium events that on occasion would migrate across the neocortex. On many occasions, activity started at the entorhinal cortex, which then propagated to the neocortex or hippocampus; however, we also observed activity starting at the anterior cortical areas closer to the medial occipital cortex. Our data are consistent with an earlier imaging study from the neonatal brain that found that cortical waves typically originate from the entorhinal cortex (Namiki et al., 2013). We caution that our work was performed on acute slices that have many severed connections between regions; thus, in an intact brain, additional regions might also be hyperexcitable in the absence of KCNQ2 channels.

KCNQ2-containing channels have a critical role in controlling the excitability of the neonatal brain (Peters et al., 2005). Immature neurons have a depolarized membrane potential that would drive many voltage-gated potassium channels to either fast or slow inactivation, rendering them incapable of clamping down the membrane potential below the threshold to generate an action potential and preventing sodium channel inactivation (Telezhkin et al., 2018). KCNQ2 channels are slow-activating, non-inactivating potassium channels (Jentsch, 2000). Thus, KCNQ2 channels would remain open at resting membrane potentials of immature neurons $(\sim-30 \mathrm{mV}$ to $-50 \mathrm{mV}$ depending on the age of the neuron) preventing unwanted excitation (Telezhkin et al., 2018). Additionally, the probability of KCNQ2 opening and voltage activation mid-point is modulated by the phospholipid PIP2 (Kim et al., 2016; Greene and Hoshi, 2017). This allows neurons to tailor KCNQ2 activity by changing membrane PIP2 levels. Thus, KCNQ2 channel gating properties and the subcellular localization, which early in development is both somatic and axonal, are suited for regulating the properties of immature neurons, before the full expression of additional voltage-gated and leak potassium channels. 
A
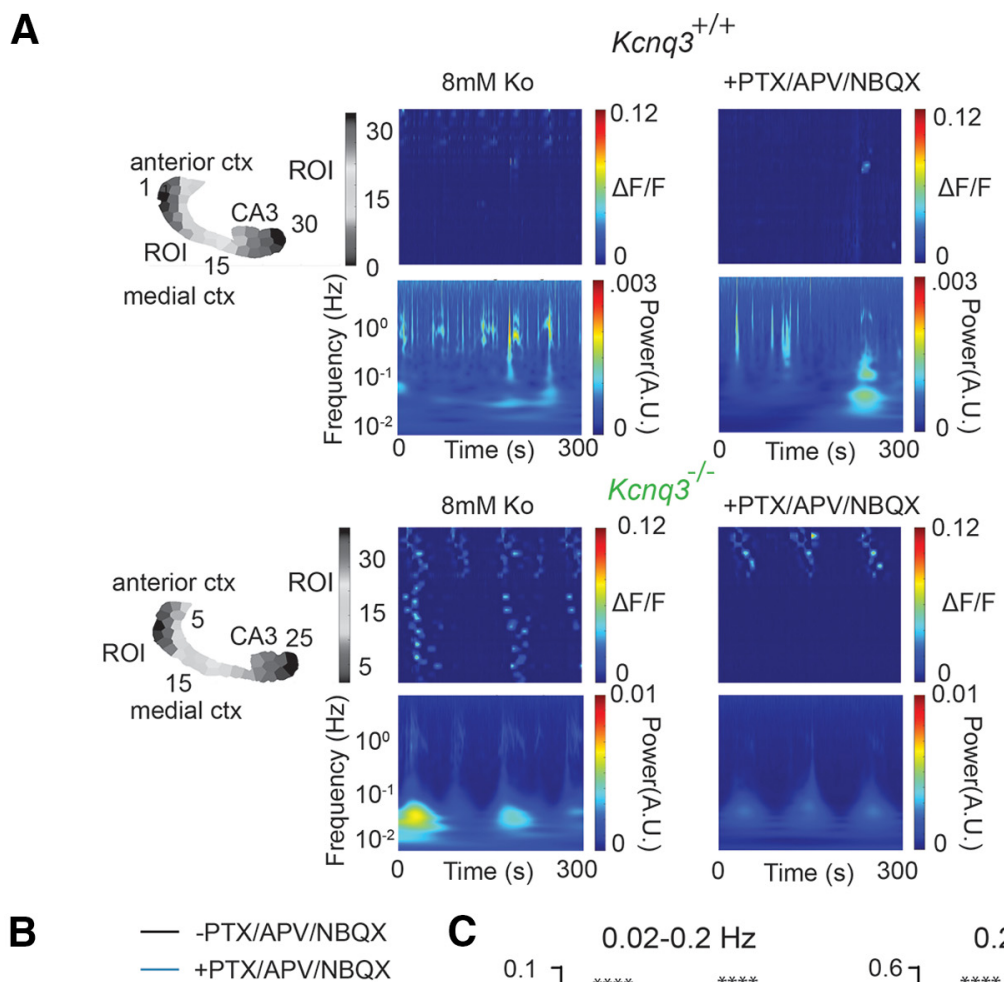

C
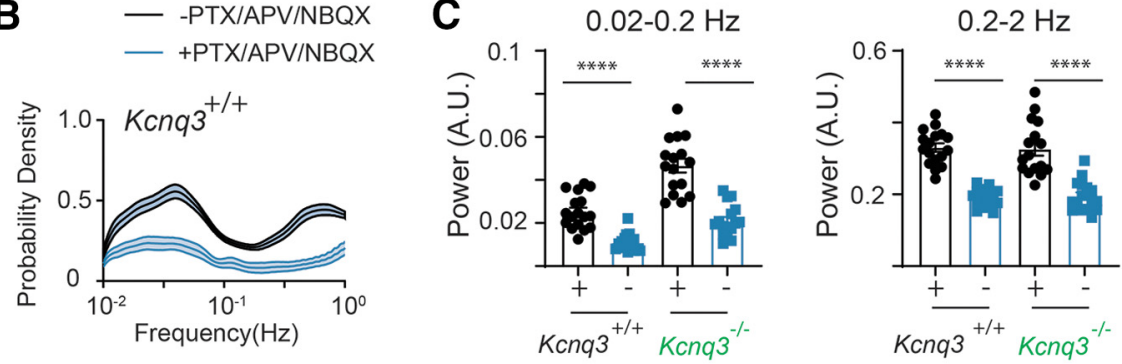

D
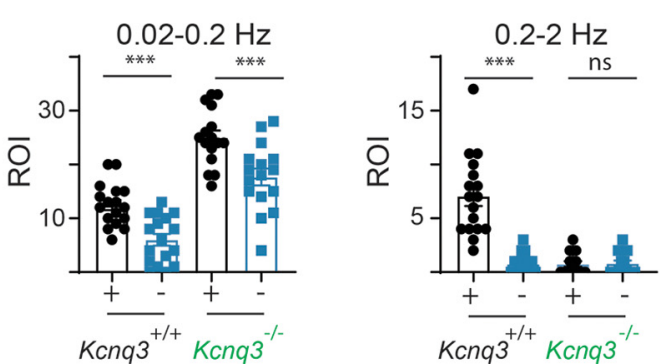

Figure 13. In $\mathrm{Kcnq}^{-1-}$ slices synaptic blockers substantially reduce slow calcium oscillations in the neocortex. All recordings were in the presence of $8 \mathrm{~mm}$ Ko. A, Representative examples of control and $K \mathrm{cnq}^{-1-} 2 \mathrm{D}$ plots along with their corresponding wavelets in the presence and absence of $50 \mu \mathrm{M}$ PTX, $25 \mu \mathrm{M}$ D-APV, and $25 \mu \mathrm{m}$ NBQX (PTX/APV/NBQX). B, Comparison of the probability density of the frequency $f$ of transient oscillations before and after addition of PTX/APV/NBQX in $K c n q 3^{+/+}(n=18)$ and $K c n q 3^{-}$ $(n=18)$ hemispheres. Note that application of the synaptic blockers did not prevent the occurrence of the slow calcium oscillations in Kcnq3-null slices. $\boldsymbol{C}$, Summary graphs show the effect of PTX/APV/NBQX on the power for the LF $(0.02-0.2 \mathrm{~Hz})$ and $\mathrm{HF}(0.2-2 \mathrm{~Hz})$ domains in $\mathrm{Kcnq}^{+/+}$and $\mathrm{Kcnq}^{-/-}$hemispheres. Note that synaptic blockers reduce the power across the board, consistent with the large reduction of the activity in the neocortex. $\boldsymbol{D}$, Summary graphs show the effect of PTX/APV/NBQX on the number of ROls in $\mathrm{KCnq}^{+/+}$and $\mathrm{Kcnq}^{-/-}$hemispheres that showed sustained oscillations at different frequencies. Data are presented as mean \pm SEM $\left({ }^{* \star *} p<0.01,{ }^{* * \star *} p<0.001\right)$. Additional details on the statistical analysis for panels $\boldsymbol{C}, \boldsymbol{D}$ are found in Table 1 under the Figure 13 section.

\section{KCNQ2 versus KCNQ3 in neonatal excitability}

Recent studies have shown that complete loss of KCNQ3 channel function can lead to pharmaco-dependent epilepsy and intellectual disability (Lauritano et al., 2019). Consistent with this, we found that loss of KCNQ3 activity leads to a hyperexcitable forebrain in the presence of an increased extracellular potassium concentration. Our data are consistent with earlier reports demonstrating mRNA expression of KCNQ3 from early developmental points and protein expression of KCNQ3 in human patients during pregestational time periods (Kanaumi et al., 2008). However, KCNQ3 loss had less robust effects than 
loss of KCNQ2 channels from the neocortex. This difference could be because of lower protein expression levels of KCNQ3 than KCNQ2 channels early in development or because of the presence of KCNQ2 channels, which could still function in the absence of KCNQ3 in neurons (Soh et al., 2014).

We also found that in Kcnq3-ablated mice, the aberrant neocortical activity was driven by synaptic activity, unlike the hypersynchrony because of KCNQ2 loss, further suggesting that loss of KCNQ2 or KCNQ3 lead to distinct effects in the developing brain. One possibility for the difference between the Kcnq3-deficient and Kcnq2-deficient neocortex might be that $K c n q 3$ was removed from both interneurons and pyramidal neurons in contrast to Kcnq2. However, we do not think this could fully explain the difference between the mouse lines as the hyperexcitability in hippocampus was insensitive to synaptic transmission blockers in both Kcnq2 and Kcnq3 transgenic animals. Rather, the differences might reflect varying KCNQ2 and KCNQ3 protein expression level among the different regions early in development. Future studies are needed to decipher the role of KCNQ2 and KCNQ3 channels in the neonatal brain.

\section{Possible model to explain our data}

What could drive the slow hypersynchronous events? Based on knowledge from previous studies we propose the following working model. Under elevated excitability conditions such as in the presence of $8 \mathrm{~mm}$ extracellular potassium, a concentration that the brain could easily reach during robust activity or seizures, Kcnq2-null and (to a lesser extent) Kcnq3-null excitatory neurons would fire a barrage of action potentials or drive neurons to a prolonged afterdepolarization because of unabated persistent sodium channel activity. A prolonged somatic and axonal depolarization in the absence of synaptic blockers might further build up extracellular potassium because of the continual activation of voltage-gated potassium channels and the engagement of glutamate-gated NMDA and AMPA receptors. The large build-up of potassium would diffuse across neighboring regions, initiating waves of depolarization and network activity across the forebrain with its scope limited by ongoing GABAA receptor activity. Additional experiments are needed to test the different scenarios using additional transgenic mice.

In conclusion, our study fills an important knowledge gap by showing that loss of KCNQ2 and to a lesser extent KCNQ3 function from the neonatal forebrain can lead to a hypersynchrony in the neonatal brain that does not fully rely on the presence of fast glutamatergic transmission.

\section{References}

Achanta R, Shaji A, Smith K, Lucchi A, Fua P, Süsstrunk S (2012) SLIC superpixels compared to state-of-the-art superpixel methods. IEEE Trans Pattern Anal Mach Intell 34:2274-2282.

Aiba I, Noebels JL (2021) Kcnq2/Kv7.2 controls the threshold and bihemispheric symmetry of cortical spreading depolarization. Brain. Advance online publication. Retrieved Mar 26, 2021. doi: 10.1093/ brain/awab141.
Carver CM, Shapiro MS (2019) Gq-coupled muscarinic receptor enhancement of KCNQ2/3 channels and activation of TRPC channels in multimodal control of excitability in dentate gyrus granule cells. J Neurosci 39:1566-1587.

Che A, Babij R, lannone AF, Fetcho RN, Ferrer M, Liston C, Fishell G, De Marco García NV (2018) Layer I interneurons sharpen sensory maps during neonatal development. Neuron 99:98-116.e7.

Cooper EC (2011) Made for "anchorin": Kv7.2/7.3 (KCNQ2/KCNQ3) channels and the modulation of neuronal excitability in vertebrate axons. Semin Cell Dev Biol 22:185-192.

Cooper EC, Harrington E, Jan YN, Jan LY (2001) M channel KCNQ2 subunits are localized to key sites for control of neuronal network oscillations and synchronization in mouse brain. $\mathrm{J}$ Neurosci 21:9529-9540.

Cornet MC, Sands TT, Cilio MR (2018) Neonatal epilepsies: clinical management. Semin Fetal Neonatal Med 23:204-212.

Garaschuk O, Linn J, Eilers J, Konnerth A (2000) Large-scale oscillatory calcium waves in the immature cortex. Nat Neurosci 3:452459.

Gee JM, Smith NA, Fernandez FR, Economo MN, Brunert D, Rothermel M, Morris SC, Talbot A, Palumbos S, Ichida JM, Shepherd JD, West PJ, Wachowiak M, Capecchi MR, Wilcox KS, White JA, Tvrdik $P(2014)$ Imaging activity in neurons and glia with a Polr2a-based and cre-dependent GCaMP5G-IRES-tdTomato reporter mouse. Neuron 83:1058-1072.

Geisheker MR, Heymann G, Wang T, Coe BP, Turner TN, Stessman HAF, Hoekzema K, Kvarnung M, Shaw M, Friend K, Liebelt J, Barnett C, Thompson EM, Haan E, Guo H, Anderlid BM, Nordgren A, Lindstrand A, Vandeweyer G, Alberti A, et al. (2017) Hotspots of missense mutation identify neurodevelopmental disorder genes and functional domains. Nat Neurosci 20:1043-1051.

Gorski JA, Talley T, Qiu M, Puelles L, Rubenstein JL, Jones KR (2002) Cortical excitatory neurons and glia, but not GABAergic neurons, are produced in the Emx1-expressing lineage. J Neurosci 22:6309-6314.

Greene DL, Hoshi N (2017) Modulation of Kv7 channels and excitability in the brain. Cell Mol Life Sci 74:495-508.

Jentsch TJ (2000) Neuronal KCNQ potassium channels: physiology and role in disease. Nat Rev Neurosci 1:21-30.

Kanaumi T, Takashima S, Iwasaki H, Itoh M, Mitsudome A, Hirose S (2008) Developmental changes in KCNQ2 and KCNQ3 expression in human brain: possible contribution to the age-dependent etiology of benign familial neonatal convulsions. Brain Dev 30:362369.

Kim KS, Duignan KM, Hawryluk JM, Soh H, Tzingounis AV (2016) The voltage activation of cortical KCNQ channels depends on global PIP2 levels. Biophys J 110:1089-1098.

Kirmse K, Kummer M, Kovalchuk Y, Witte OW, Garaschuk O, Holthoff K (2015) GABA depolarizes immature neurons and inhibits network activity in the neonatal neocortex in vivo. Nat Commun 6:7750.

Lauritano A, Moutton S, Longobardi E, Tran Mau-Them F, Laudati G, Nappi P, Soldovieri MV, Ambrosino P, Cataldi M, Jouan T, Lehalle D, Maurey H, Philippe C, Miceli F, Vitobello A, Taglialatela M (2019) A novel homozygous KCNQ3 loss-of-function variant causes nonsyndromic intellectual disability and neonatal-onset pharmacodependent epilepsy. Epilepsia Open 4:464-475.

Murata Y, Colonnese MT (2020) GABAergic interneurons excite neonatal hippocampus in vivo. Sci Adv 6:eaba1430.

Namiki S, Norimoto H, Kobayashi C, Nakatani K, Matsuki N, Ikegaya Y (2013) Layer III neurons control synchronized waves in the immature cerebral cortex. J Neurosci 33:987-1001.

Nappi P, Miceli F, Soldovieri MV, Ambrosino P, Barrese V, Taglialatela M (2020) Epileptic channelopathies caused by neuronal Kv7 (KCNQ) channel dysfunction. Pflugers Arch 472:881-898.

Okada M, Zhu G, Hirose S, Ito KI, Murakami T, Wakui M, Kaneko S (2003) Age-dependent modulation of hippocampal excitability by KCNQ-channels. Epilepsy Res 53:81-94.

Peters HC, Hu H, Pongs O, Storm JF, Isbrandt D (2005) Conditional transgenic suppression of $\mathrm{M}$ channels in mouse brain reveals 
functions in neuronal excitability, resonance and behavior. Nat Neurosci 8:51-60.

Qiu C, Johnson BN, Tallent MK (2007) K+ M-current regulates the transition to seizures in immature and adult hippocampus. Epilepsia 48:2047-2058.

Safiulina VF, Zacchi P, Taglialatela M, Yaari Y, Cherubini E (2008) Low expression of Kv7/M channels facilitates intrinsic and network bursting in the developing rat hippocampus. J Physiol 586:54375453.

Satterstrom FK, Kosmicki JA, Wang J, Breen MS, De Rubeis S, An JY, Peng M, Collins R, Grove J, Klei L, Stevens C, Reichert J, Mulhern MS, Artomov M, Gerges S, Sheppard B, Xu X, Bhaduri A, Norman U, Brand $\mathrm{H}$, et al. (2020) Large-scale exome sequencing study implicates both developmental and functional changes in the neurobiology of autism. Cell 180:568-584.e23.

Sheroziya MG, von Bohlen Und Halbach O, Unsicker K, Egorov AV (2009) Spontaneous bursting activity in the developing entorhinal cortex. J Neurosci 29:12131-12144.

Singh NA, Otto JF, Dahle EJ, Pappas C, Leslie JD, Vilaythong A, Noebels JL, White HS, Wilcox KS, Leppert MF (2008) Mouse models of human KCNQ2 and KCNQ3 mutations for benign familial neonatal convulsions show seizures and neuronal plasticity without synaptic reorganization. J Physiol 586:3405-3423.

Sipila ST, Huttu K, Soltesz I, Voipio J, Kaila K (2005) Depolarizing GABA acts on intrinsically bursting pyramidal neurons to drive giant depolarizing potentials in the immature hippocampus. $J$ Neurosci 25:5280-5289.

Soh H, Pant R, LoTurco JJ, Tzingounis AV (2014) Conditional deletions of epilepsy-associated KCNQ2 and KCNQ3 channels from cerebral cortex cause differential effects on neuronal excitability. $J$ Neurosci 34:5311-5321.
Soh H, Park S, Ryan K, Springer K, Maheshwari A, Tzingounis AV (2018) Deletion of KCNQ2/3 potassium channels from PV+ interneurons leads to homeostatic potentiation of excitatory transmission. Elife 7:e38617.

Sood D, Cairns DM, Dabbi JM, Ramakrishnan C, Deisseroth K, Black LD 3rd, Santaniello S, Kaplan DL (2019) Functional maturation of human neural stem cells in a 3D bioengineered brain model enriched with fetal brain-derived matrix. Sci Rep 9:17874.

Telezhkin V, Straccia M, Yarova P, Pardo M, Yung S, Vinh NN, Hancock JM, Barriga GG, Brown DA, Rosser AE, Brown JT, Canals JM, Randall AD, Allen ND, Kemp PJ (2018) Kv7 channels are upregulated during striatal neuron development and promote maturation of human iPSC-derived neurons. Pflugers Arch 470:1359-1376.

Traynelis SF, Dingledine R (1988) Potassium-induced spontaneous electrographic seizures in the rat hippocampal slice. $J$ Neurophysiol 59:259-276.

Verneuil J, Brocard C, Trouplin V, Villard L, Peyronnet-Roux J, Brocard F (2020) The M-current works in tandem with the persistent sodium current to set the speed of locomotion. PLoS Biol 18: e3000738.

Wang T, Hoekzema K, Vecchio D, Wu H, Sulovari A, Coe BP, Gillentine MA, Wilfert AB, Perez-Jurado LA, Kvarnung M, Sleyp Y, Earl RK, Rosenfeld JA, Geisheker MR, Han L, Du B, Barnett C, Thompson E, Shaw M, Carroll R, et al. (2020) Large-scale targeted sequencing identifies risk genes for neurodevelopmental disorders. Nat Commun 11:4932.

Watanabe H, Nagata E, Kosakai A, Nakamura M, Yokoyama M, Tanaka K, Sasai H (2000) Disruption of the epilepsy KCNQ2 gene results in neural hyperexcitability. J Neurochem 75:28-33. 